\title{
Rapid global path planning algorithm for unmanned surface vehicles in large-scale and multi-island marine environments
}

\author{
Dong Wang ${ }^{1,2}$, Jie Zhang ${ }^{\text {Corresp., } 1,2}$, Jiucai Jin ${ }^{\text {Corresp., }}{ }^{\text {, Deqing Liu }}{ }^{2}$, Xingpeng Mao ${ }^{1}$ \\ ${ }^{1}$ School of Electronics and Information Engineering, Harbin Institute of Technology, Harbin, Heilongjiang, China \\ 2 First Institute of Oceanography, Ministry of Natural Resources, Qingdao, Shandong, China \\ Corresponding Authors: Jie Zhang, Jiucai Jin \\ Email address: zhangjie@fio.org.cn, jinjiucai@fio.org.cn
}

A global path planning algorithm for unmanned surface vehicles (USVs) with short time requirements in large-scale and complex multi-island marine environments is proposed. The fast marching method-based path planning for USVs is performed on grid maps, resulting in a decrease in computer efficiency for larger maps. This can be mitigated by improving the algorithm process. In the proposed algorithm, path planning is performed twice in maps with different spatial resolution (SR) grids. The first path planning is performed in a low SR grid map to determine effective regions, and the second is executed in a high SR grid map to rapidly acquire the final high precision global path. In each path planning process, a modified inshore-distance-constraint fast marching square (IDC-FM ${ }^{2}$ ) method is applied. Based on this method, the path portions around an obstacle can be constrained within a region determined by two inshore-distance parameters. The path planning results show that the proposed algorithm can generate smooth and safe global paths wherein the portions that bypass obstacles can be flexibly modified. Compared with the path planning based on the IDC-FM ${ }^{2}$ method applied to a single grid map, this algorithm can significantly improve the calculation efficiency while maintaining the precision of the planned path. 


\section{Rapid global path planning algorithm for unmanned surface 2 vehicles in large-scale and multi-island marine environments}

3 Dong Wang ${ }^{1,2}$, Jie Zhang ${ }^{1,2}$, Jiucai Jin², Deqing Liư², Xingpeng Mao ${ }^{1}$

$4{ }^{1}$ School of Electronics and Information Engineering, Harbin Institute of Technology, Harbin,

5 Heilongjiang, China

$6 \quad 2$ First Institute of Oceanography, Ministry of Natural Resources, Qingdao, Shandong, China

Corresponding Author:

9 Jie Zhang ${ }^{1,2}$, Jiucai Jin ${ }^{2}$

No. 6 Xianxialing Road, Laoshan District, Qingdao, Shandong, 266061, China

Email address: zhangjie@fio.org.cn, jinjiucai@fio.org.cn

\section{Abstract}

A global path planning algorithm for unmanned surface vehicles (USVs) with short time requirements in large-scale and complex multi-island marine environments is proposed. The fast marching method-based path planning for USVs is performed on grid maps, resulting in a decrease in computer efficiency for larger maps. This can be mitigated by improving the algorithm process. In the proposed algorithm, path planning is performed twice in maps with different spatial resolution (SR) grids. The first path planning is performed in a low SR grid map to determine effective regions, and the second is executed in a high SR grid map to rapidly acquire the final high precision global path. In each path planning process, a modified inshoredistance-constraint fast marching square (IDC-FM²) method is applied. Based on this method, the path portions around an obstacle can be constrained within a region determined by two inshore-distance parameters. The path planning results show that the proposed algorithm can generate smooth and safe global paths wherein the portions that bypass obstacles can be flexibly modified. Compared with the path planning based on the IDC-FM ${ }^{2}$ method applied to a single grid map, this algorithm can significantly improve the calculation efficiency while maintaining the precision of the planned path.

\section{Introduction}

Research on unmanned surface vehicles (USVs) has received increased attention in various military and civilian applications over recent years (Yan et al., 2010; Campbell, Naeem \& Irwin, 2012; Liu et al., 2016). Robust and reliable navigation, guidance, and control (NGC) systems are required for USVs to perform a variety of complex marine missions. Path planning is an essential 
35

36

37

component of an NGC system. The main aim of path planning is to calculate a collision-free path with specific requirements. It determines the automation level of USVs and ensures the reliability and success of missions (Tan et al., 2020).

Global path planning is an important aspect of path planning. A variety of algorithms for the global path planning of USVs have been studied for different requirements. Dijkstra's algorithm (Dijkstra, 1959) is a classic graph-based algorithm that can plan the shortest global path for USVs (Xie et al., 2016; Singh et al., 2018). As an improvement of Dijkstra's algorithm, the A* algorithm (Hart, Nilsson \& Raphael, 1968) and its related improved algorithms are also commonly used in the global path planning of USVs (Campbell, Naeem \& Irwin, 2012), such as the direction priority sequential selection method (Naeem, Irwin \& Yang, 2012); the A* algorithm, which considers environment effects (EEA*) (Lee et al., 2105); and the ARC-Theta* algorithm, which considers both the angular rate and heading angle of USVs (Kim et al., 2014). These algorithms exhibit acceptable convergence and consistency; however, the planned paths must be further smoothed. The artificial potential field (APF) method (Khatib, 1986) is a classic method for both global and local path planning. The main drawback of the traditional APF method is the local minimum problem. Therefore, different studies (Guo, Gao \& Cui, 2013; Song, Hao \& Su, 2020) have been conducted to solve the local minimum problem for the path planning of robots or USVs. Evolutionary methods such as the genetic algorithm (GA) (Kim et al., 2017; Arzamendia et al., 2019; Wang et al., 2020), particle swarm optimization (PSO) (Song et al., 2015), and ant colony optimization (ACO) (Song, 2014; Xia et al., 2019) have also been applied in the path planning of USVs. GA is robust and adaptable, but it has the shortcomings of poor local search ability and the premature convergence phenomenon. Therefore, other methods, such as the simulated annealing algorithm, are usually introduced to improve the performance of GA (Zhang, Xu \& Xie, 2019). Nevertheless, the path planned by GA still lacks consistency. Similar to GA, PSO has the advantage of strong robustness and the shortcoming of premature convergence. However, it is dependent on various parameters, and there is no specific theory for guiding the setting of these parameters for different problems. Similarly, the parameter selection of ACO is more dependent on experience, and this algorithm can easily fall into local extremum.

In some special applications, the shortest time may be an important requirement for USVs. The fast marching method (FMM) can be a solution for time-optimal global path planning. This method was first proposed by Tsitsiklis (1995), and Adalsteinsson \& Sethian (1995) independently and was extended by Sethian (1999). The path planned by the FMM is usually extremely close to the obstacles. One solution is to adjust the speed map, as exemplified by the method with an adjusted cost function (Messias et al., 2014) and the $\mathrm{FM}^{2}$ method (Garrido et al., 2007). FMM-based methods have been widely used in path planning applications (Gómez et al., 2013; Amorim \& Ventura, 2014; Alvarez et al., 2015; González et al., 2016). Marine applications based on FMM were introduced by Garrido, Alvarez \& Moreno (2020). Interesting modifications for the $\mathrm{FM}^{2}$ method have been performed, and the FMM has been subjected to a vector field considering the effects of several vector variables such as wind flow or water currents (Garrido, Alvarez \& Moreno, 2020). In addition, studies on path following and obstacle 
avoidance and formations have also been conducted using FMM (Garrido, Alvarez \& Moreno, 2020). USV formation path planning has also been performed by Liu \& Bucknall (2015) and Tan et al. (2020), and an angle-guidance $\mathrm{FM}^{2}$ method has been used for the Springer USV to make the generated path compliant with the dynamics and orientation restrictions of USVs ( Liu \& Bucknall, 2016; Liu, Bucknall \& Zhang, 2017). An improved anisotropic fast marching method using a multi-layered fast marching was proposed by Song, Liu \& Bucknall (2017), which combines different environmental factors and provides interesting results. In addition to the global path planning, the FMM-based methods also show potential in collision avoidance of USVs (Wang, Jin \& Er, 2019; Garrido, Alvarez \& Moreno, 2020). These successful studies have demonstrated the potential of FMM-based methods in global path planning of USVs.

The basic FMM can plan time-optimal paths for USVs. However, some of the main shortcomings is that the paths planned by the FMM are too close to obstacles, and there may be abrupt turns when the paths bypass obstacles with sharp corners. Thus, the $\mathrm{FM}^{2}$ method is proposed to address these problems, and two dimensionless parameters are introduced to adjust the paths more flexibly (Garrido et, al. ,2007; Garrido, Alvarez \& Moreno, 2020). However, the two introduced parameters lack clear physical meaning, and the suitable adjustment is difficult to identify with specific values. Moreover, the adjustment effects of these two parameters are not common because the adjustment degree with the same parameters varies with different grid maps. Another common problem about FM-based methods is that the computational efficiency of path planning decreases sharply when the scale of the grid map is very large. Therefore, we make two improvements to address the mentioned shortcomings of the basic $\mathrm{FM}^{2}$ method. First, we introduced an inshore-distance-constraint fast marching square (IDC-FM²) method to improve the inshore path adjustment performance for the first time. Comparing with the basic $\mathrm{FM}^{2}$ method, the IDC-FM ${ }^{2}$ method applies two inshore distance parameters other than the two dimensionless parameters to adjust the paths around the obstacles. The adjustment effects for path planning of the IDC-FM² method are stable in different grid maps as the IDC-FM ${ }^{2}$ method can constrain the path portions around the obstacles within the region constrained by the two inshore distance parameters. Further, to improve the computational efficiency, the algorithm that applies the IDC-FM² method based on two-level spatial resolution grid maps is designed.

\section{Related Methods}

\section{Environment map model}

In 2D global path planning applications based on the FMM or its improved methods, discrete numerical calculations are based on cartesian grid maps. Therefore, an environment map should first be converted into a binary grid map with a suitable spatial resolution (SR). Free and opensource satellite images (such as Google satellite images) can be used as the data source for the maps in most USV applications, and the corresponding grid maps can be generated using image processing (Shi et al., 2018) combined with manual assistance. The binary grid is set as an obstacle grid when an obstacle exists at the geographic location ( 0 values); otherwise, it is set as a free grid (1 value). 


\section{Fast marching square method}

115 When using FM-based methods to plan a path, the basis is the FMM. The core work of the FMM 116 is calculating solutions of the Eikonal equation (Tsitsiklis, 1995; Adalsteinsson \& Sethian, 1995).

117 The Eikonal equation describes a wave front propagation scenario from sources with the speed of 118 a wave front given as $F_{x}$ at cell $x$. It can be expressed as $\left\|\nabla T_{x}\right\| F_{x}=1$, where $T_{x}$ is the arrival 119 time of the wave from the source to the cell $x$ and $\nabla$ is a vector differential operator. From the perspective of time-cost, the Eikonal equation can be expressed in another form (that of Lin,

121 2003):

$122\left\|\nabla T_{x}\right\|=\tau_{x}$

123 where $\tau_{x}$ is the time-cost at cell $x$ and is equivalent to $1 / F_{x}$. The solution we want to calculate is $124 T_{x}$, and all of them compose the arrival time map, $\boldsymbol{T}(x)$. All time-cost $\tau_{x}$ compose the time-cost 125 function map, $\boldsymbol{\tau}(x)$.

126 The FMM was first proposed independently by Tsitsiklis (1995) and Adalsteinsson \& Sethian 127 (1995).The solution $T_{x}$ in cell $x$ can be interpreted as the wave arrival time from the nearest 128 source to cell $x$. Three cell sets (the accepted set $\boldsymbol{\delta}_{\mathrm{A}}$, trial set $\boldsymbol{S}_{\mathrm{T}}$, and far set $\boldsymbol{S}_{\mathrm{F}}$ ) are defined to 129 calculate $\boldsymbol{T}(x)$. The set of all cells at which $T_{x}$ will not change is $\boldsymbol{\delta}_{\mathrm{A}} \cdot \boldsymbol{S}_{\mathrm{T}}$ is the set of cells to be 130 examined. Every $T_{x}$ in $\boldsymbol{S}_{\mathrm{T}}$ has been previously computed but may be updated. $\boldsymbol{S}_{\mathrm{F}}$ is the set of all 131 other cells with $T_{x}$, which has never been computed (Lin, 2003).

The calculation process includes initialization and loop procedures. During initialization, source cells $x_{0}$ with $T_{x_{0}}=0$ are set to $\boldsymbol{\delta}_{\mathrm{T}}$, and all other cells are set to $\boldsymbol{\delta}_{\mathrm{F}}$ with $T_{x}=\infty$. After the initialization, the cell $x_{m}$ with the smallest $T$ value in $\boldsymbol{S}_{\mathrm{T}}$ is selected and moved from $\boldsymbol{\delta}_{\mathrm{T}}$ to $\boldsymbol{\delta}_{\mathrm{A}}$. Thereafter, the non-accepted neighbor cells of $x_{m}$ are updated, including the solutions and cell sets. Considering Tsitsiklis's deduction (Tsitsiklis, 1995; Lin, 2003), each of the new neighbor 137 solutions is determined by:

$138 T_{x}=\min \left(\tilde{T}_{x},\left(T_{x_{m} x_{i}}, i=1,2\right)\right)$ where $\tilde{T}_{x}$ denotes the original solution. $T_{x_{m} x_{i}}{ }^{i}=1,2$ are the candidate solutions for the paths that pass through the line segment $x_{m} x_{i}$ and propagate to cell $x$ (see Fig. 1), which are calculated by:

$141 T_{x_{m} x_{i}}=\left\{\begin{array}{cc}\frac{1}{2}\left(T_{x_{m}}+T_{x_{i}}+\sqrt{2 \tau_{x}^{2}-\left(T_{x_{m}}-T_{x_{i}}\right)^{2}}\right) & , T_{x_{m} x_{i}}>T_{x_{m},} \text {,and } T_{x_{m} x_{i}}>T_{x_{i}} \\ \min \left(T_{x_{m}}, T_{x_{i}}\right)+\tau_{x} & \text {,others }\end{array}\right.$

142 where $T_{x_{m}}$ and $T_{x_{i}}$ are the accepted solutions at cells $x_{m}$ and $x_{i}$, respectively. If cell $x_{i}$ is in $\boldsymbol{S}_{\mathrm{T}}$, $143 T_{x_{i}}$ is $\infty$. For the cell set, if a non-accepted neighbor cell $x$ is in $\boldsymbol{S}_{\mathrm{F}}$, it moves from $\boldsymbol{S}_{\mathrm{F}}$ to $\boldsymbol{S}_{\mathrm{T}}$. The 
144 loop procedure is performed continually until all cells are in $\boldsymbol{S}_{\mathrm{A}}$. The resulting map is the arrival 145 time map, $\boldsymbol{T}$.

146 The main disadvantage of the basic FMM is that the computed path is too close to obstacles 147 and forces the vehicle to perform abrupt turns (Garrido, Alvarez \& Moreno, 2020). As described 148 in Garrido et, al.(2007) and Garrido, Alvarez \& Moreno, (2020), a smooth path with sufficient 149 safety distances from obstacles can be computed using the $\mathrm{FM}^{2}$ method. This method applies the 150 basic FMM twice. The procedure for computing paths is described as follows (Garrido, Alvarez 151 \& Moreno, 2020):

152 1. The environment is modeled as a binary grid map (Fig. 2A).

153 2. The FM- ${ }^{\text {st }}$ step. All obstacle cells are used as wave sources $(T=0)$, expanding several waves at the same time at a constant speed. The value of each cell in the resulting map indicates the time required for a wave to reach the closest obstacle (see Fig. 2B). This is proportional to the distance from the obstacles. By reversing the meaning of these values, they can be understood as the maximum admissible speed at each cell. Finally, the speed values are rescaled to fix a maximum cell value of 1 .

Modifications of the speed map with two parameters, $\alpha$ and $\beta$, can adjust distances

160

161 between the computed paths and obstacles. The value of each cell in the speed map $F_{i, j}$ is adjusted exponentially by $\alpha$ :

164

165

166

167

168

169

170

171

172

173

174

175

The parameter $\beta$ is used to saturate the values in the speed map. It is defined within the range of 0 and 1 . Every $F_{i, j}$ with a value greater than $\beta$ is set to one (see Fig. $2 C$ ).

Comparing with a path without modifications, the path modified by $\alpha$ will be closer to obstacles when $\alpha<1$. On the contrary, if $\alpha>1$, the modified path will stay further away from obstacles. The parameter $\beta$ allows the path to move closer to obstacles. When $\beta$ is smaller, the modified path will be closer to obstacles.

3. The FM-2nd step. The goal point is used as a unique wave source. The wave is expanded over the map until the starting point is reached. The speed at each cell (equivalent to $1 / \tau_{x}$ ) is obtained from the modified speed map computed in the FM- $1^{\text {st }}$ step. The resulting arrival time map is shown in Fig. $2 D$.

4. Finally, a gradient descent is applied over the resulting arrival time map from the starting point to the goal point. An optimal path in terms of the arrival time, smoothness, and safety is obtained.

\section{Gradient descent method}

The global path can be extracted by applying the gradient descent method. The path propagates along the gradient descent direction from the starting position $P_{\mathrm{s}}$ to the goal position $P_{\mathrm{g}}$ with a step length $d$. The value of $d$ can be set to a value equal to the SR of the grid map. The gradient of the cell $x_{i, j}$ is calculated by: 
$181 \nabla T_{i, j}=\left[\begin{array}{ll}\frac{T_{i+1, j}-T_{i-1, j}}{2} & \frac{T_{i, j+1}-T_{i, j-1}}{2}\end{array}\right]^{\mathrm{T}}$

182 where $T_{i, j}$ represents the arrival time value at grid $x_{i, j}$ and the non-italic $\mathrm{T}$ in the upper-right

183 corner represents the transpose of vector $\left[\begin{array}{ll}x & y\end{array}\right]$.

184 The path waypoints are not limited to cell centers. Generally, the gradient of a path waypoint 185 $P_{n}=(u, v)$ located in the cell $x_{i, j}$ is approximated by the cell gradient $\nabla T_{i, j}$. However, a modified 186 gradient $\nabla T_{u, v}$ calculated by the bilinear interpolation can be used to improve the path precision 187 (see Fig. 3).

\section{Proposed algorithm}

189

190

191

192

193

194

195

196

197

198

199

200

201

To improve the computational efficiency of global path planning in a large-scale and multiisland marine environment, the proposed algorithm performs path planning twice for different purposes. First, the path planning is performed in a low SR (LSR) grid map to determine an effective region. The final global path is then obtained in the second path planning within the effective region of a high SR (HSR) grid map. The relevant methods and procedures applied in the algorithm are as follows. The complete algorithm flow is summarized in the last part of this section.

\section{Mapping of two-level SR grid maps}

The two-level SR grid maps are contained in the HSR and LSR grid maps. The HSR grid map is directly obtained from the Google satellite images data. A mapping relationship between the LSR cells and the corresponding $L \times L$ HSR cell sub-blocks is established, which can be expressed as:

$\left(i_{\mathrm{L}}, j_{\mathrm{L}}\right) \sim\left[\begin{array}{ccc}\left(i_{\mathrm{H}}^{\prime}, j_{\mathrm{H}}^{\prime}+L-1\right) & \cdots & \left(i_{\mathrm{H}}^{\prime}+L-1, j_{\mathrm{H}}^{\prime}+L-1\right) \\ \vdots & \ddots & \vdots \\ \left(i_{\mathrm{H}}^{\prime}, j_{\mathrm{H}}^{\prime}\right) & \cdots & \left(i_{\mathrm{H}}^{\prime}+L-1, j_{\mathrm{H}}^{\prime}\right)\end{array}\right]$

202 where $\left(i_{\mathrm{L}}, j_{\mathrm{L}}\right)$ are the LSR cell coordinates, and $\left(i_{\mathrm{H}}^{\prime}, j_{\mathrm{H}}^{\prime}\right)$ are the original cell coordinates of the 203 mapped HSR cell sub-block, as shown in Fig. 4.

204 To map the LSR grid map to a sub-block of the HSR grid map, the following is used:

$205\left\{\begin{array}{l}i_{\mathrm{H}}^{\prime}=i_{\mathrm{Ho}}+L i_{\mathrm{L}} \\ j_{\mathrm{H}}^{\prime}=j_{\mathrm{Ho}}+L j_{\mathrm{L}}\end{array}\right.$

206 To map from a sub-block of the HSR grid map to a cell of the LSR grid map, we use:

$207\left\{\begin{array}{l}i_{\mathrm{L}}=f_{\text {floor }}\left(\frac{i_{\mathrm{H}}-i_{\mathrm{Ho}}}{L}\right) \\ j_{\mathrm{L}}=f_{\text {floor }}\left(\frac{j_{\mathrm{H}}-j_{\mathrm{Ho}}}{L}\right)\end{array}\right.$ 
208 where $\left(i_{\mathrm{H}}, j_{\mathrm{H}}\right), i_{\mathrm{H}}^{\prime} \leq i_{\mathrm{H}} \leq i_{\mathrm{H}}^{\prime}+L-1, j_{\mathrm{H}}^{\prime} \leq j_{\mathrm{H}} \leq j_{\mathrm{H}}^{\prime}+L-1$ are the HSR cell coordinates. $\left(i_{\mathrm{Ho}}, j_{\mathrm{Ho}}\right)$ 209 are the original cell coordinates of the original HSR cell sub-block (see Fig. 4), which are 210 determined by:

$211\left\{\begin{array}{l}i_{\mathrm{Ho}}=\left(i_{\mathrm{Hg}}-f_{\text {floor }}\left(\frac{L}{2}\right)\right) \% L \\ j_{\mathrm{Ho}}=\left(j_{\mathrm{Hg}}-f_{\text {floor }}\left(\frac{L}{2}\right)\right) \% L\end{array}\right.$

212 where $\left(i_{\mathrm{Hg}} j_{\mathrm{Hg}}\right)$ are the HSR cell coordinates of the goal cell $x_{\mathrm{g}}$, the function $f_{\text {floor }}(m)$ indicates 213 a rounding down of $m$, and $a \% b$ indicates the modulo operation.

214 The SR of the LSR grid map is:

$215 \quad D_{\text {LRes }}=L D_{\text {HRes }}$

216 where $D_{\text {HRes }}$ is the SR of the HSR grid map. In general, $L$ is defined as:

$217 L \leq f_{\text {round }}\left(\frac{D_{\mathrm{Th}}}{2 D_{\mathrm{HReS}}}\right)$

218 where $D_{\mathrm{Th}}$ is the distance threshold of the virtual obstacle influence, and the function $f_{\text {round }}(m)$ 219 indicates a rounding of $m$. The cell numbers of the LSR grid map in the X-axis and Y-axis 220 directions are:

$221 M_{\mathrm{L}}=f_{\text {floor }}\left(\frac{M_{\mathrm{H}}-i_{\mathrm{Ho}}}{L}\right)$

$222 N_{\mathrm{L}}=f_{\text {floor }}\left(\frac{N_{\mathrm{H}}-j_{\mathrm{Ho}}}{L}\right)$

223 where $M_{\mathrm{H}}$ and $N_{\mathrm{H}}$ are the cell numbers of the HSR grid map in the $\mathrm{X}$-axis and $\mathrm{Y}$-axis directions, 224 respectively.

225 Based on the mapping relationship, an LSR cell type is determined by comparing the setting 226 threshold $\Gamma$ and obstacle cell proportion $\gamma$ in the HSR cell sub-block. If $\gamma>\Gamma$, the LSR cell is set 227 as an obstacle cell; otherwise, it is set as a free cell. $\Gamma$ prefers to select a small value to retain as 228 much obstacle information as possible.

\section{IDC-FM $^{2}$ method}

230 In the basic $\mathrm{FM}^{2}$ method, the path is improved using a modified speed map. Two parameters, $\alpha$ 231 and $\beta$, are used to modify the speed map. These modifications ensure the flexibility of the 232 computed path. However, these two parameters have no clear physical meaning. The 233 normalization of the speed values in the FM-1 ${ }^{\text {st }}$ step also results in several shortcomings. For 234 example, all the speed values in the speed map must be calculated. When the map range changes 235 slightly, the entire speed map rescales and differs in the same locations of the original map, and 236 the parameters must be adjusted. 
237

238

239

240

241

242

243

244

245

246

247

248

249

250

251

252

253

254

255

256

257

258

259

260

261

262

263

264 Therefore, $w_{x}$ with respect to $D_{x}$ is used to approximate $w_{x}$ : constrained in a region determined by $D_{\mathrm{Th}}$ and $D_{\mathrm{sc}}$.

\section{Time-cost weighting function} weighting function map, $\boldsymbol{W}^{\prime}$, is designed as:

$w_{x}^{\prime}=\left\{\begin{array}{cc}\infty & , T_{x}=0 \\ 1+a\left(\frac{T_{\mathrm{Th}}}{T_{x}}-1\right)^{b} & , T_{x}>0\end{array}\right.$ positive coefficients to be determined. where $D_{x}$ is the closest distance to obstacles), then:

$D_{x} \approx D_{\operatorname{Res}} \frac{T_{x}}{\tau}$

$265 w_{x}\left(D_{x}\right)=\left\{\begin{array}{cc}\infty & , D_{x}=0 \\ 1+a\left(\frac{D_{\mathrm{Th}}}{D_{x}}-1\right)^{b} & , 0<D_{x} \leq D_{\mathrm{Th}} \\ 1 & , D_{x}>D_{\mathrm{Th}}\end{array}\right.$

An IDC-FM ${ }^{2}$ method is proposed to address these shortcomings. In this method, a time-cost weighting function, $w_{x}$, is introduced to adjust the speed map. Three inshore-distance parameters (the distance threshold $D_{\mathrm{Th}}$, as well as the virtual strong and weak constraint distance parameters, $D_{\mathrm{sc}}$ and $D_{\mathrm{wc}}$, respectively) and two weighting function values with respect to $D_{\mathrm{sc}}$ and $D_{\mathrm{wc}}\left(w_{\mathrm{sc}}\right.$ and $w_{\mathrm{wc}}$, respectively) are used to determine the function $w_{x}$, and $D_{\mathrm{Th}}$ is used to achieve the same function as the speed saturation. Although five parameters are set, only two inshoredistance parameters, $D_{\mathrm{Th}}$ and $D_{\mathrm{sc}}$, must be considered, while suitable values of $w_{\mathrm{sc}}$ and $w_{\mathrm{wc}}$ can be selected as constant values. Based on this method, the path portion around an obstacle is

The time-cost weighting function $w_{x}$ is introduced as a concept of the virtual obstacle influence.

When a USV is far away from obstacles, there is no obstacle influence. As the USV approaches, the virtual obstacle influence increases slowly at first and the amplitude increases gradually. The obstacle influence increases dramatically when the USV is close to within a certain degree.

Similar to the FM-1 ${ }^{\text {st }}$ step in the basic FM $^{2}$ method, all obstacle cells are set as source cells to first calculate the arrival time map. The difference here is that a threshold $T_{\mathrm{Th}}=\tau{ }^{D_{\mathrm{Th}}} / D_{\mathrm{Res}}$ could be set to speed up the calculation, where $D_{\text {Res }}$ is the SR of the map, and $\tau$ is the unified time-cost which can take 1 as the value. In the loop procedure, if the smallest $T$ value of a cell in $\boldsymbol{S}_{\mathrm{T}}$ is larger than or equal to $T_{\mathrm{Th}}$, all $T$ values of non-accepted cells can be set as $T_{\mathrm{Th}}$ directly. Based on the resulting arrival time map, $\boldsymbol{T}_{\text {sat }}$, with a saturation threshold, the time-cost

where $w_{x}^{\prime}$ and $T_{x}$ are the values of the cell $x$ in $\boldsymbol{W}^{\prime}$ and $\boldsymbol{T}_{\text {sat }}$, respectively, and $a$ and $b$ are two

When a free grid, $x$, is within the virtual influenced scope of obstacles (i.e., $0<D_{x} \leq D_{\mathrm{Th}}$, 
266 where $w_{x}\left(D_{x}\right)$ is the value in cell $x$ of the approximate time-cost weighting function map, $\boldsymbol{W}$.

267 The time-cost value of each grid in the time-cost map, which is the inversion of the speed map, is 268 then adjusted to:

$269 \operatorname{new} \tau_{x}=w_{x}\left(D_{x}\right) \tau_{x}$

270 Eq. (16) implies that $w_{x}$ increases when grid $x$ is closer to obstacles, and it also increases

271 when $D_{x}$ is small. The planned path tends to select points with a lower arrival time-cost.

272 Therefore, when the path is close to obstacles, it will be farther away from obstacles compared to 273 the path planned by the basic FMM.

\section{Parameters for the time-cost weighting function}

275 Five parameters, $D_{\mathrm{Th}}, D_{\mathrm{sc}}, D_{\mathrm{wc}}, w_{\mathrm{sc}}$, and $w_{\mathrm{wc}}$, are introduced to determine the coefficients $a$ and $276 b$, which are determined by:

$277 \quad b=\frac{\ln \left(w_{\mathrm{sc}}-1\right)-\ln \left(w_{\mathrm{wc}}-1\right)}{\ln \left(1-e_{\mathrm{sc}}\right)-\ln \left(1-e_{\mathrm{wc}}\right)+\ln e_{\mathrm{wc}}-\ln e_{\mathrm{sc}}}$

$278 \quad a=\left(w_{\mathrm{sc}}-1\right)\left(\frac{e_{\mathrm{sc}}}{1-e_{\mathrm{sc}}}\right)^{b}$

279 where $e_{\mathrm{sc}}=D_{\mathrm{sc}} / D_{\mathrm{Th}}, e_{\mathrm{wc}}=D_{\mathrm{wc}} / D_{\mathrm{Th}}, D_{\mathrm{sc}}<D_{\mathrm{wc}}<D_{\mathrm{Th}}$, and $w_{\mathrm{sc}}>w_{\mathrm{wc}}>1$.

280 The parameter $D_{\mathrm{Th}}$ determines the largest virtual influence scope of the obstacles. The

281 suggestion for the selection of $D_{\mathrm{Th}}$ is that there should be a sufficiently safe buffer area for

282 obstacles. The parameter $D_{\text {sc }}$ is very important for the safety of the USV. First, it is suggested

283 that this parameter satisfies:

284

$D_{\mathrm{sc}} \geq v_{\mathrm{U}, \max } t_{\mathrm{r}}-\frac{v_{\mathrm{U}, \max }^{2}}{2 a_{\mathrm{d}}}$

285 where $v_{\mathrm{U}, \max }$ is the maximum speed of the USV, $t_{\mathrm{r}}$ is the reaction time of the thruster, and $a_{\mathrm{d}}$ is

286 the negative maximum acceleration of the USV under braking. In an unknown environment, it is 287 better to select a slightly larger value to ensure safety, which is similar in environments with 288 shallow and reef waters. This value may be relatively small in deep and reef-free waters to 289 improve path efficiency. $D_{\text {wc }}$ is a parameter that acts as the adjusted constraint distance. In a 290 non-channel ocean area, the path portion around the obstacles will be limited to the region 291 between $D_{\mathrm{wc}}$ and $D_{\mathrm{Th}}$. The $D_{\mathrm{wc}}$ value can be set independently or determined jointly by $D_{\mathrm{Th}}$ and $292 D_{\text {sc }}$. In this study, it is set as:

293

$D_{\mathrm{wc}}=D_{\mathrm{Th}}-\frac{\sqrt{2}}{2}\left(D_{\mathrm{Th}}-D_{\mathrm{sc}}\right)$

294 The area around the obstacles is divided into four parts by the three inshore-distance

295 parameters: $D_{\mathrm{sc}}, D_{\mathrm{wc}}$, and $D_{\mathrm{Th}}$. The three parts extending from an obstacle to the outside region 296 are the danger region, $S_{\mathrm{d}}$; the strong constraint region, $S_{\mathrm{sc}}$; and the weak constraint region, $S_{\mathrm{wc}}$ 
297 (see Fig. 5). The influences of $w_{\mathrm{wc}}$ and $w_{\mathrm{sc}}$ on the path are shown in Fig. 5. As shown in Fig.

$2985 A$, the path will be located far from the obstacle until it is near the boundary of $S_{\mathrm{wc}}$ when $w_{\mathrm{wc}}$ is 299 large. In contrast, the path is closer to an obstacle when $w_{\text {sc }}$ is large (see Fig. 5B). The minimum 300 degree of the path close to $S_{\mathrm{sc}}$ is mainly determined by $w_{\mathrm{wc}}$, which can be inferred by further 301 comparing the paths in Fig. $5 B$ with the closest path to the obstacle $\left(w_{\text {wc }}=1.1\right)$ in Fig. $5 \mathrm{~A}$.

302 However, regardless of the change in $w_{\mathrm{wc}}$ and $w_{\mathrm{sc}}\left(w_{\mathrm{sc}}>w_{\mathrm{wc}}>1\right)$, the path portions bypassing 303 obstacles will always be within $S_{\mathrm{wc}}$ or around the outside boundary of $S_{\mathrm{wc}}$ in non-channel ocean 304 areas. In channel areas where there is no $S_{\mathrm{wc}}$, the path will follow the quasi-centerline of the 305 channel if it passes through the channel. In this study, the values of $w_{\mathrm{sc}}=40.0$ and $w_{\mathrm{wc}}=2.0$ 306 were selected and fixed. The paths can be flexibly modified by the inshore-distance parameters $307 D_{\mathrm{Th}}$ and $D_{\text {sc }}$.

\section{Determination of effective regions within the HSR grid map}

309 The effective region within the HSR grid map is important for improving the computational 310 efficiency of the proposed algorithm. It is acquired based on the mapping between the LSR and 311 HSR grid maps when the corresponding region within the LSR grid map is determined.

312 Two effective regions that are respectively used in the FM- $1^{\text {st }}$ and FM-2 $2^{\text {nd }}$ steps of the IDC$313 \mathrm{FM}^{2}$ method must be determined. The low-precision initial path, $\ell_{\text {ini }}$, is obtained first based on 314 the LSR arrival time map $\boldsymbol{T}_{\text {L }}$. Two effective regions within the LSR grid map, $S_{\text {LER_1st }}$ and $315 S_{\text {LER_2nd }}$, are determined by expanding the grids around the "path-passed" grids of $\ell_{\text {ini }}$. As shown 316 in Fig. 6, the nearest neighbor grid among the four neighbor cells of the waypoint $P_{i}=\left(x_{i}, y_{i}\right)$ is 317 defined as the "path-passed" grid. When all "path-passed" grids are determined, the $\kappa_{2 \text { nd }}=\kappa$ 318 levels of the cells are extended to obtain $S_{\text {LER_2nd }}$. Considering $S_{\text {LER_1st }}$, there are three different 319 situations:

320 - Situation 1: there is no cell with $w_{x}>1$ in $S_{\text {LER_2nd }}$;

321 - Situation 2: there is at least one cell with $w_{x}>1$ and no obstacle cell in $S_{\text {LER_2nd }}$.

322 - Situation 3: there is at least one obstacle cell in $S_{\text {LER_2nd }}$.

323 In Situations 1 and 3, $\kappa_{1 s t}=\kappa$. In Situation 2, $S_{\text {LER_2nd }}$ is extended continuously with $\Delta \kappa$ 324 levels of cells until there is at least one obstacle cell for the first time, and $\kappa_{1 \mathrm{st}}=\kappa+\Delta \kappa$. Finally, 325 two corresponding effective regions within the HSR grid map, $S_{\text {HER_1st }}$ and $S_{\text {HER_2nd }}$, are 326 determined and used in the different FM steps of the IDC-FM² method to obtain the HSR arrival 327 time map $\boldsymbol{T}_{\mathrm{H}}$.

328 The extended parameter $\kappa$ affects the paths (recorded as $\ell_{\kappa}$ ) based on the proposed algorithm. 329 When $\kappa$ is not adequately large, these paths may not be consistent with the reference path $\ell_{\text {ref }}$, 330 which is acquired by applying the IDC-FMㄹ method in the HSR grid map directly. A navigable 331 global path is used as a case to compare the consistency between $\ell_{\kappa}$ and $\ell_{\text {ref }}$. The basic 
332 parameters used in the proposed algorithm are listed in Table 1. Using $\ell_{\text {ref }}$ as the reference, the

333 distances between the corresponding waypoints of $\ell_{\kappa}(\kappa=3, \ldots, 6)$ and $\ell_{\text {ref }}$ are shown in Fig. 7. It

334 is shown that the largest distance decreases when $\kappa$ increases, and the distance becomes 0 when

$335 \quad \kappa \geq 7$. Other cases were tested and similar results were obtained. These results indicate that there

336 is good consistency when $\kappa$ is sufficiently large (such as $\kappa=10$ ). $\Delta \kappa$ is a variable parameter that

337 was determined based on three different cases.

\section{Algorithm flow}

339

340

341

342

343

344

345

346

347

348

349

350

351

352

353

354

355

356

357

358

359

360

361

362

363

364

365

366

367

The proposed algorithm is improved based on the basic $\mathrm{FM}^{2}$-based algorithm. The basic FM²based algorithm is executed directly on a single grid map. The algorithm flow of this basic algorithm is shown in Fig. 8 in the form of main data stream and used methods. This algorithm flow has two main steps. In Step S1, an arrival time map with obstacle sources and a saturation threshold, $\boldsymbol{\mathcal { T }}_{\text {sat, }}$, is obtained by first applying the FMM. Then, the approximate time-cost weighting function map, $\boldsymbol{W}$, is calculated and used to adjust the time-cost map. Based on the adjusted time-cost map, an arrival time map with the goal point source, $\mathcal{T}$, is obtained by applying the FMM again. The entire process is used to obtain $\mathcal{T}$ by applying the IDC-FM ${ }^{2}$ method. Finally, the global path is acquired based on $\mathcal{T}$ by applying the gradient descent method in Step S2.

The main data stream in the proposed algorithm and the methods used to obtain them are shown in Fig. 9. The main flow is as follows:

1. Step T1. Obtain the two-level SR grid maps;

2. Step T2. Obtain the LSR arrival time map $\boldsymbol{T}_{\mathrm{L}}$ in the LSR grid map;

3. Step T3. Determine the effective regions within the HSR grid map $\left(S_{\text {HER_1st }}\right.$ and $\left.S_{\text {HER_2nd }}\right)$;

4. Step T4. Obtain the HSR grid arrival time map $\boldsymbol{T}_{\mathrm{H}}$ with the effective region constraint in both FM steps of the IDC-FM ${ }^{2}$ method. There are several adjustments in both FM steps compared to the basic FMM performance. For the FM- $1^{\text {st }}$ step, all $w_{x}$ values within $S_{\text {HER_1st }}$ need not be adjusted, and this step can be ignored in Situation 1. In Situation 2 and Situation 3, all free cells out of $S_{\text {HER_1st }}$ are set to $\boldsymbol{S}_{\mathrm{A}}$ with $T=\infty$ in the initialization of this step. In the FM-2 ${ }^{\text {nd }}$ step, all free cells out of $S_{\text {HER_2nd }}$ are set to $\boldsymbol{S}_{\mathrm{A}}$ with $T=\infty$ in the initialization;

5. Step T5. Acquire the final high precision global path by applying the gradient descent method based on $\boldsymbol{T}_{\mathrm{H}}$.

\section{Results and discussion}

\section{Simulation environments}

Two surrounding spatial areas of Zhucha Island and Changhai County were selected as the simulation environments. The original maps adopted Google satellite images, as shown in Fig. 10. The spatial resolutions in the longitude and latitude directions are approximately $4.8 \mathrm{~m}$ and

Peer] Comput. Sci. reviewing PDF | (CS-2021:02:58122:2:0:NEW 27 May 2021) 
$3683.9 \mathrm{~m}$, respectively. Temporary binary grid maps are obtained based on the image processing

369

370

371

372

373

374

375

376

377

378

379

380

381

382

383

384

385

386

387

388

389

390

391

392

393

394

395

396

397

398

399

400

401

402

method (Shi et al., 2018) combined with manual assistance, and then the HSR grid maps with $D_{\text {HRes }}=10 \mathrm{~m}$ in both the longitude and latitude directions are determined by resampling processing. The corresponding binary grid maps are presented in Fig. 11. Their ranges are $7 \mathrm{~km} \times 7 \mathrm{~km}$ and $64 \mathrm{~km} \times 48 \mathrm{~km}$, respectively.

\section{Inshore-distance-constraint performances}

A path planning from $P_{\mathrm{s}}=[4.3 \mathrm{~km}, 2.8 \mathrm{~km}]$ to $P_{\mathrm{g}}=[3.6 \mathrm{~km}, 2.0 \mathrm{~km}]$ is used as a typical case to analyze the inshore-distance-constraint performance of the IDC-FM ${ }^{2}$ method using the inshoredistance parameters $D_{\mathrm{Th}}$ and $D_{\mathrm{sc}}$. As shown in Fig. 12, the path planned based on the basic $\mathrm{FMM}, \ell_{\mathrm{FMM}}$, is very close to the islands when this path bypasses them. For comparison, all paths planned by the proposed algorithm, $\ell_{1}$ to $\ell_{4}$, are located away from islands by a certain distance. They are therefore significantly better choices from a safety perspective.

Paths $\ell_{1}$ to $\ell_{4}$ are acquired using different inshore-constraint distance parameters $\left(D_{\mathrm{Th}}\right.$ and $D_{\mathrm{sc}}$ , see Table 2 wherein $D_{\mathrm{wc}}$ is calculated based on $D_{\mathrm{Th}}$ and $D_{\mathrm{sc}}$ ) in the IDC-FM ${ }^{2}$ method in which $w_{\mathrm{sc}}=40.0$ and $w_{\mathrm{wc}}=2.0$. These paths are clearly adjusted by these distance parameters. When the distance constraints $\left(D_{\mathrm{Th}}\right.$ and $D_{\mathrm{sc}}$ ) are small, the path (such as $\ell_{1}$, see Fig. 12) will be a somewhat close to the islands. The estimated quasi-closest distance from $\ell_{1}$ to an island is approximately $38 \mathrm{~m}$, which is shorter than half of the shortest channel width $\left(d_{\mathrm{hscw}} \approx 101 \mathrm{~m}\right)$. In this situation, $\ell_{1}$ is always outside of its $S_{\mathrm{sc}}(<28.2 \mathrm{~m})$ value, and the path portions around the islands are located in the region of its $S_{\mathrm{wc}}(28.2 \mathrm{~m}$ to $60.0 \mathrm{~m})$. When the distance constraints increase, the paths (such as $\ell_{2}$ and $\ell_{3}$, see Fig. 12) in the non-channel areas will be outside of $S_{\text {sc }}$. The estimated quasi-closest distances (approximately $115 \mathrm{~m}$ and $128 \mathrm{~m}$, respectively) were larger than $d_{\text {hscw }}$. However, $\ell_{2}$ and $\ell_{3}$ will be along the quasi-midline of the channel in the channel area, regardless of whether $d_{\mathrm{hscw}}$ is greater or less than $D_{\mathrm{wc}}$ for these two paths because the weighting time-cost will be smaller than the path around the islands (as in $\ell_{4}$, see Fig. 12). If the distance constraints are further increased, $\ell_{4}$ will be selected for safety, although more time will be required. These results indicate that the path based on the proposed algorithm can be adjusted by setting different inshore-constraint distance parameters to meet safety requirements. Additionally, when the inshore-constraint distance parameters are determined, the path will cross a channel when its width is adequately large; otherwise, the path will bypass around the islands.

One classical approach to plan collision-free paths is applying the FMM within the map with inflated obstacles. For comparison, paths planned by the classical approach and the IDC-FM ${ }^{2}$ method are shown in Fig. 13. The starting and goal positions are $P_{\mathrm{s}}=[4.15 \mathrm{~km}, 2.74 \mathrm{~km}]$ and $P_{\mathrm{g}}$ $=[4.33 \mathrm{~km}, 2.40 \mathrm{~km}]$, and the island obstacles have been inflated by $94.0 \mathrm{~m}$. As shown in Fig. 13 , the path planned by the classical approach, $\ell_{\mathrm{FMM}}+$ inflated is obviously influenced by the 
403 shape of the inflated obstacle. In some path turns which are marked by ellipses in Fig. 13, they 404 are both affected by the shape of the inflated obstacle and the time-value gradient calculation of 405 cells adjacent to the inflated obstacle. A smooth path, $\ell_{\mathrm{FMM}}+$ inflated + smooth, , is also shown in Fig. 406 13. Although it removes abrupt turn waypoints, the path is still not very smooth. As 407 comparisons, paths planed based on the IDC-FMㄹ method, $\ell_{1}$ and $\ell_{2}$, are obviously smoother 408 than $\ell_{\mathrm{FMM}}+$ inflated + smooth. . In addition, when using the proposed rapid path planning algorithm 409 based on two-level SR grid maps to improve the computational efficiency, the channel will be 410 mapped as obstacle cells when mapping the HSR grid map to the LSR grid map in this case 411 (taking the mapping parameter $L=8$ as shown in Table 1), because of the influence of the 412 inflated obstacle. This unexpected mapping will result in the loss of the path through the channel.

\section{Global path planning in a large-scale and complex multi-island environment}

414 To verify the path planning ability and the computational efficiency improvement of the 415 proposed algorithm in a large-scale and relatively complex multi-island environment, the 416 simulation environment shown in Fig. $11 \mathrm{~B}$ is selected, and the long-length path cases of USVs 417 around the islands or across channels are investigated.

418 Path planning cases

419 Five typical path cases were selected to demonstrate the performance of the proposed algorithm. 420 The $P_{\mathrm{s}}$ and $P_{\mathrm{g}}$ groups are listed in Table 3 . When determining the inshore-distance parameter $D_{\mathrm{sc}}$ 421 , a USV with $v_{\mathrm{U}, \max }=6 \mathrm{~m} / \mathrm{s}, t_{\mathrm{r}}=2 \mathrm{~s}, a_{\mathrm{d}}=-1 \mathrm{~m} / \mathrm{s}^{2}$ is considered as a case. Based on Eq. (20), $422 D_{\mathrm{sc}}$ is suggested to select a value larger than $30 \mathrm{~m}$. Therefore, a larger value $D_{\mathrm{sc}}=50 \mathrm{~m}$ is used 423 for the path planning for the selected five path cases. Another inshore-distance parameter $D_{\mathrm{Th}}$ $424=200 \mathrm{~m}$ is selected empirically. The main parameters used in these path planning cases are 425 shown in Table 1.

426 As shown in Fig. 14, all paths successfully bypass the islands. The enlarged views (see Fig. 42715 , every path is displayed with a solid line) provide further details. Every path maintains a 428 relatively safe distance when close to an island, and smooth when turning around the island. For 429 a narrow channel of a certain degree (usually wider than $2 D_{\text {LRes }}$ ), the path is planned along the 430 quasi-midline of the channel to ensure that it is as safety as possible. The path lengths range from 431 about $26.52 \mathrm{~km}$ to $47.86 \mathrm{~km}$ (see Table 3). These lengths can cover the range of most 432 applications of current small-and medium-sized USVs. These results show the effective path 433 planning ability of the proposed algorithm in a large-scale and complex multi-island 434 environment.

\section{Computational efficiency improvement}

436 To verify the computational efficiency improvement of the proposed method, the time spent on 437 the five typical paths (see Table 3) based on the proposed algorithm was calculated. For 438 comparison, the time spent on the basic algorithm which is executed directly on a single HSR 439 grid map by applying the IDC-FM ${ }^{2}$ method was also calculated as a reference. The $\mathrm{C}$ algorithm 
440 code was tested on a computer with a Core i5-6300U CPU and 8G memory, which runs a 64-bit 441 Win7 operating system.

442 Path planning for every typical path was repeated 10 times. Every planning time starts from 443 the HSR grid map reading (in Steps S1 and T1) and ends when the global path has been 444 calculated (in Steps S2 and T5). Because the path planning is performed on a Windows operating 445 system, which involves multitasking, there may be many factors influencing the planning time,

446

447

448

449

450

451

452

453

454

455

456

457

458

459

460

461

462

463

464

465

466

467

468

469

470

471

472

473

474

\section{Conclusions} algorithm. T4-T5. such as the variable CPU usage, random CPU hit rate to the cache, and possible thread scheduling. Therefore, the average planning time is used to evaluate the computational efficiency. The average planning time results of the five planned paths are presented in Table 4. These results indicate that the computational efficiency of the proposed algorithm based on twolevel grid maps is significantly higher than the algorithm based on a single HSR grid map. The time for all cases is approximately $2 \mathrm{~s}$, indicating that this method can be used in less demanding real-time planning applications. This can effectively improve the practicality of the proposed

When planning a path by applying the basic $\mathrm{FM}^{2}$-based algorithm, if the grid map scale is very large, two aspects of calculations will severely increase compared with small-scale maps. First, as the number of cells increases significantly, the calculations for free cells increase. Second, $\boldsymbol{\delta}_{\mathrm{T}}$ changes in every iteration of calculations. The overall scale of $\boldsymbol{S}_{\mathrm{T}}$ clearly increases, resulting in the increase in sorting time of the adopted priority heap struct. This compared algorithm, whose algorithm flow is shown in Fig. 8, is used directly on a single HSR grid map. The valid scale of the HSR grid map is the number of free cells. For comparison, the proposed algorithm determines the effective regions within the HSR grid map first and then plans a path within the determined effective regions. As shown in Fig. 9, Steps T1-T3 complete the determination of effective regions, and Steps T4-T5 realize the path planning. The same function of the path planning is also realized by Steps $\mathrm{S} 1-\mathrm{S} 2$, as shown in Fig. 8. Comparing the path planning steps of the compared and proposed algorithms, the numbers of free cells in effective regions are much less than the ones in the HSR grid map. This indicates that the calculations for free cells and the overall scale of $\boldsymbol{S}_{\mathrm{T}}$ will greatly decrease. Therefore, the planning time of the proposed algorithm reduces. Certainly, there are more steps in the proposed algorithm. Planning time spent on these steps (Steps T1-T3) mainly depends on the LSR mapping parameter $L$. When the planning time spent on the compared algorithm, $t_{\text {ref }}$, is large enough, the planning time spent on Step T2 can reduce to less than $t_{\text {ref }} / L^{2}$ (in the cases shown in Table 4 , this value is approximately $t_{\text {ref }} /\left(2 L^{2}\right)$ ).

The planning time spent on Steps T1 and T3 is often much less than $t_{\text {ref }}$. Therefore, the increased planning time spent on Steps T1-T3 is much less than the saved planning time spent on Steps 
476 In some special USV applications such as sea rescue, it requires the USV to reach a goal position

477 as soon as possible. FMM is a suitable global path planning method which can plan a time-

478 optimal global path. However, the planned paths are not safe enough when they bypass

479 obstacles, because of that they are too close to the obstacles. Therefore, FMM should be

480 improved for safety considerations. One classical approach is applying the FMM within the map

481 with inflated obstacles. Although the planned paths are safe by inflating the obstacle size, there

482 may be abrupt turns when the obstacles have sharp corners, and the planned paths are influenced

483 by shapes of inflated obstacles and may be not very smooth. Such paths are usually unfriendly

484 for USVs. A rapid global path planning algorithm applying an IDC-FM² method in two-level SR

485 grid maps is proposed for USV applications with short time requirements in large-scale and

486 complex multi-island environments. This algorithm can acquire a continuous, smooth, quasi-

487 time-optimal path while maintaining a safe distance around obstacles when bypassing obstacles.

488 When the path is near obstacles, it is limited to a safe area determined by two inshore-distance

489 parameters. By adjusting these two inshore-distance parameters, the path can be modified

490 flexibly. Although the time optimality is missed by using the IDC-FM ${ }^{2}$ method, the safety with a

491 higher priority in most applications has been ensured initially while the optimal loss of time

492 remains at a certain level. The two-path planning process based on two-level SR grid maps

493 improves the computational efficiency compared with the basic FM²-based method. The

494 planning time on the order of seconds is acceptable in many global path planning applications of

495 USVs. Meanwhile, the planning time of this order of magnitude is typically short enough for

496 USVs in most situations with the requirement to replan the path. This indicates the potential of

497 replanning by using the proposed algorithm from the perspective of planning time. As a

498 comparison, when using the classical approach which applies the FMM within the map with

499 inflated obstacles, narrow channels will be easier to map as obstacles when mapping the HSR

500 grid map to the LSR grid map in the rapid planning process based on two-level SR grid maps.

501 This shortcoming may result in the loss of some possible paths through channels.

502 On the other hand, there are still some challenges when using the proposed algorithm. For

503 example, how to accurately obtain the location information of newly detected obstacles and how

504 to add this information into the grid map when replanning are the common challenges. The IDC-

$505 \mathrm{FM}^{2}$ method also needs to be modified to plan the path meeting the dynamic characteristics of a

506 USV. Otherwise, the USV may not follow the replanned path successfully at the beginning of the 507 path.

508 One shortcoming of the proposed algorithm is that paths through channels that are very narrow 509 but still passable in reality may be missed because of the first path planning process in the LSR 510 grid map. The introduction of environmental effects into the proposed algorithm is an important 511 task to be performed in future works. Marine experiments should also be conducted to verify the 512 validity of the algorithm.

513

\section{Acknowledgments}

515 We would like to thank Editage (www.editage.cn) for English language editing. 
516

517

518

519

520

521

522

523

524

525

526

527

528

529

530

531

532

533

534

535

536

537

538

539

540

541

542

543

544

545

546

547

548

549

550

551

552

553

554

555

556

557

558

559

560

561

\section{References}

Adalsteinsson D, and Sethian JA. 1995. A fast level set method for propagating interfaces. Journal of Computational Physics 118:269-277. 10.1006/jcph.1995.1098

Alvarez D, Gómez J, Garrido S, and Moreno L. 2015. 3D robot formations path planning with fast marching square. Journal of Intelligent \& Robotic Systems 80:507-523. 10.1007/s10846-015$0187-1$

Amorim D, and Ventura R. 2014. Towards efficient path planning of a mobile robot on rough terrain. 2014 IEEE International Conference on Autonomous Robot Systems and Competitions (ICARSC). Espinho, Portugal: IEEE. p 22-27.

Arzamendia M, Gregor D, Reina DG, and Toral SL. 2019. An evolutionary approach to constrained path planning of an autonomous surface vehicle for maximizing the covered area of Ypacarai Lake. Soft Computing 23:1723-1734. 10.1007/s00500-017-2895-x

Campbell S, Naeem W, and Irwin GW. 2012. A review on improving the autonomy of unmanned surface vehicles through intelligent collision avoidance manoeuvres. Annual Reviews in Control 36:267283. 10.1016/j.arcontrol.2012.09.008

Dijkstra EW. 1959. Communication with an automatic computer doctor PhD thesis. University of Amsterdam.

Gómez JV, Alvarez D, Garrido S, and Moreno L. 2019. Fast methods for Eikonal equations: an experimental survey. IEEE Access 7:39005-39029. 10.1109/ACCESS.2019.2906782

Gómez JV, Lumbier A, Garrido S, and Moreno L. 2013. Planning robot formations with fast marching square including uncertainty conditions. Robotics and Autonomous Systems 61:137-152. https://doi.org/10.1016/j.robot.2012.10.009

Garrido S, Alvarez D, and Moreno LE. 2020. Marine applications of the fast marching method. Frontiers in Robotics and AI 7:1-12. 10.3389/frobt.2020.00002

Garrido S, Moreno L, Blanco D, and Martin F. 2007. FM2: A real-time fast marching sensor-based motion planner. 2007 IEEE/ASME international conference on advanced intelligent mechatronics. Zurich, Switzerland: IEEE. p 1-6.

González V, Monje CA, Moreno L, and Balaguer C. 2016. Fast marching square method for UAVs mission planning with consideration of Dubins model constraints. IFAC-PapersOnLine 49. 10.1016/j.ifacol.2016.09.029

Guo J, Gao Y, and Cui G. 2013. Path planning of mobile robot based on improved potential field. Information Technology Journal 12:2188-2194. 10.3923/itj.2013.2188.2194

Hart PE, Nilsson NJ, and Raphael B. 1968. A formal basis for the heuristic determination of minimum cost paths. IEEE Transactions on Systems Science and Cybernetics 4:100-107. 10.1109/TSSC.1968.300136

Khatib O. 1986. Real-time obstacle avoidance for robot manipulator and mobile robots. The International Journal of Robotics Research 5:90-98. 10.1177/027836498600500106

Kim H, Kim D, Shin JU, Kim H, and Myung H. 2014. Angular rate-constrained path planning algorithm for unmanned surface vehicles. Ocean Engineering 84:37-44. 10.1016/j.oceaneng.2014.03.034 
562

563

564

565

566

567

568

569

570

571

572

573

574

575

576

577

578

579

580

581

582

583

584

585

586

587

588

589

590

591

592

593

594

595

596

597

598

599

600

601

602

603

604

605

606

607

608

609

610

611

612
Kim H, Kim SH, Jeon M, Kim J, Song S, and Paik KJ. 2017. A study on path optimization method of an unmanned surface vehicle under environmental loads using genetic algorithm. Ocean Engineering 142:616-624. 10.1016/j.oceaneng.2017.07.040

Lee T, Kim H, Chung H, Bang Y, and Myung H. 2015. Energy efficient path planning for a marine surface vehicle considering heading angle. Ocean Engineering 107:118-131. 10.1016/j.oceaneng.2015.07.030

Lin Q. 2003. Enhancement, extraction, and visualization of 3D volume data doctor PhD thesis. Linköping University.

Liu Y, and Bucknall R. 2015. Path planning algorithm for unmanned surface vehicle formations in a practical maritime environment. Ocean Engineering 97:126-144. 10.1016/j.oceaneng.2015.01.008

Liu Y, and Bucknall R. 2016. The angle guidance path planning algorithms for unmanned surface vehicle formations by using the fast marching method. Applied Ocean Research 59:327-344. 10.1016/j.apor.2016.06.013

Liu Y, Bucknall R, and Zhang X. 2017. The fast marching method based intelligent navigation of an unmanned surface vehicle. Ocean Engineering 142:363-376. 10.1016/j.oceaneng.2017.07.021

Liu Z, Zhang Y, Yu X, and Yuan C. 2016. Unmanned surface vehicles: an overview of developments and challenges. Annual Reviews in Control 41:71-93. 10.1016/j.arcontrol.2016.04.018

Messias J, Ventura R, Lima P, Sequeira J, Alvito P, Marques C, and Carriço P. 2014. A robotic platform for edutainment activities in a pediatric hospital. 2014 IEEE International Conference on Autonomous Robot Systems and Competitions (ICARSC). Espinho, Portugal: IEEE. p 193-198.

Naeem W, Irwin GW, and Yang A. 2012. COLREGs-based collision avoidance strategies for unmanned surface vehicles. Mechatronics 22:669-678. 10.1016/j.mechatronics.2011.09.012

Sethian JA. 1999. Level set methods and fast marching methods. Cambridge, UK Cambridge University Press.

Shi B, Su Y, Zhang H, Liu J, and Wan L. 2018. Obstacles modeling method in cluttered environments using satellite images and its application to path planning for USV. International Journal of Naval Architecture and Ocean Engineering 11:202-210. 10.1016/j.ijnaoe.2018.04.001

Singh Y, Sharma S, Sutton R, and Hatton D. 2018. Towards use of Dijkstra algorithm for optimal navigation of an unmanned surface vehicle in a real-time marine environment with results from artificial potential field. TransNav the International Journal on Marine Navigation and Safety of Sea Transportation 12:125-131. 10.12716/1001.12.01.14

Song C. 2014. Global path planning method for USV system based on improved ant colony algorithm. Applied Mechanics and Materials 568-570:785-788. 10.4028/www.scientific.net/AMM.568570.785

Song J, Hao C, and Su J. 2020. Path planning for unmanned surface vehicle based on predictive artificial potential field. International Journal of Advanced Robotic Systems 17:1-13. $10.1177 / 1729881420918461$

Song L, Mao Y, Xiang Z, Zhou Y, and Du K. 2015. A study on path planning algorithms based upon particle swarm optimization. Journal of Information \& Computational Science 12:673-680. 10.12733/jics20105310

Song R, Liu Y, and Bucknall R. 2017. A multi-layered fast marching method for unmanned surface vehicle path planning in a time-variant maritime environment. Ocean Engineering 129:301-317. 10.1016/j.oceaneng.2016.11.009

Tan G, Zou J, Zhuang J, Wan L, Sun H, and Sun Z. 2020. Fast marching square method based intelligent navigation of the unmanned surface vehicle swarm in restricted waters. Applied Ocean Research 95:102018. 10.1016/j.apor.2019.102018

Tsitsiklis JN. 1995. Efficient algorithms for globally optimal trajectories. IEEE Transactions on Automatic Control 40:1528-1538.

Wang N, Jin X, and Er J. 2019. A multilayer path planner for a USV under complex marine environments. Ocean Engineering 184:1-10. 10.1016/j.oceaneng.2019.05.017

Peer] Comput. Sci. reviewing PDF | (CS-2021:02:58122:2:0:NEW 27 May 2021) 
613 Wang N, Xu H, Li C, and Yin J. 2020. Hierarchical path planning of unmanned surface vehicles: a fuzzy

614

615

616

617

618

619

620

621

622

623

624

625

626 artificial potential field approach. International Journal of Fuzzy Systems. 10.1007/s40815-02000912-y

Xia G, Han Z, Zhao B, Liu C, and Wang X. 2019. Global path planning for unmanned surface vehicle based on improved quantum ant colony algorithm. Math Probl Eng 2019:1-10. $10.1155 / 2019 / 2902170$

Xie S, Wu P, Liu H, Yan P, Li X, Luo J, and Li Q. 2016. A novel method of unmanned surface vehicle autonomous cruise. Industrial Robot: An International Journal 43:121-130. 10.1108/IR-05-20150097

Yan R, Pang S, Sun H, and Pang Y. 2010. Development and missions of unmanned surface vehicle. Journal of Marine Science and Application 9:451-457. 10.1007/s11804-010-1033-2

Zhang W, Xu Y, and Xie J. 2019. Path planning of USV based on improved hybrid genetic algorithm. 2019 European Navigation Conference (ENC). Warsaw, Poland: IEEE. p 1-7. 


\section{Table 1 (on next page)}

Basic parameter values for the proposed algorithm. 
1 Table 1:

2 Basic parameter values for the proposed algorithm.

\begin{tabular}{cccccc}
\hline Parameter & Value & Parameter & Value & Parameter & Value \\
\hline$L$ & 8 & $\Gamma$ & 0.2 & $D_{\mathrm{Th}}(\mathrm{m})$ & 200 \\
$D_{\mathrm{sc}}(\mathrm{m})$ & 50 & $w_{\mathrm{sc}}$ & 40.0 & $w_{\mathrm{wc}}$ & 2.0 \\
\hline
\end{tabular}

3 


\section{Table 2 (on next page)}

Inshore-constraint distance parameters for the determination of $w_{x}$. 
1 Table 2:

2 Inshore-constraint distance parameters for the determination of $w_{x}$.

\begin{tabular}{cccc}
\hline Path & $\boldsymbol{D}_{\mathbf{T h}}(\mathbf{m})$ & $\boldsymbol{D}_{\mathbf{w c}}(\mathbf{m})$ & $\boldsymbol{D}_{\mathbf{s c}}(\mathbf{m})$ \\
\hline$\ell_{1}$ & 60.0 & 28.2 & 15.0 \\
$\ell_{2}$ & 200.0 & 79.8 & 30.0 \\
$\ell_{3}$ & 200.0 & 104.5 & 65.0 \\
$\ell_{4}$ & 200.0 & 118.7 & 85.0 \\
\hline
\end{tabular}

3 


\section{Table 3(on next page)}

Quantified information of the five typical paths. 
1 Table 3:

2 Quantified information of the five typical paths.

\begin{tabular}{cccc}
\hline Path & $\boldsymbol{P}_{\mathbf{s}}(\mathbf{k m})$ & $\boldsymbol{P}_{\mathbf{g}}(\mathbf{k m})$ & Length $(\mathbf{k m})$ \\
\hline$\ell_{1}$ & {$[35.34,39.25]$} & {$[15.31,11.65]$} & 38.84 \\
$\ell_{2}$ & {$[19.42,41.02]$} & {$[17.10,3.63]$} & 37.90 \\
$\ell_{3}$ & {$[42.96,43.67]$} & {$[46.34,8.24]$} & 38.20 \\
$\ell_{4}$ & {$[36.11,18.77]$} & {$[47.44,41.01]$} & 26.47 \\
$\ell_{5}$ & {$[3.95,26.52]$} & {$[50.45,30.83]$} & 47.82 \\
\hline
\end{tabular}

3 


\section{Table 4 (on next page)}

Planning times of five typical path cases for the compared algorithm based on a single HSR grid map and the proposed algorithm based on two-level SR grid maps. 
1 Table 4:

2 Planning times of five typical path cases for the compared algorithm based on a single HSR 3 grid map and the proposed algorithm based on two-level SR grid maps.

\begin{tabular}{ccc}
\hline \multirow{2}{*}{ Path } & \multicolumn{2}{c}{$\begin{array}{c}\text { Average planning times (s) } \\
\text { Proposed algorithm }\end{array}$} \\
\hline$\ell_{1}$ & 29.21 & 1.94 \\
$\ell_{2}$ & 24.65 & 1.95 \\
$\ell_{3}$ & 30.25 & 1.97 \\
$\ell_{4}$ & 16.44 & 1.76 \\
$\ell_{5}$ & 40.40 & 2.24 \\
\hline
\end{tabular}

4 


\section{Figure 1}

Diagram of updating the neighbor of $x_{m}$ in a 4-neighbor scheme.

Cell $x$ is the neighbor cell to be updated. In this case, $x$ is in $\mathbf{S}_{\mathrm{T}}$. If $x$ is a cell in $\boldsymbol{S}_{\mathrm{F}}$, it moves from $\boldsymbol{S}_{\mathrm{F}}$ to $\boldsymbol{S}_{\mathrm{T}}$ 


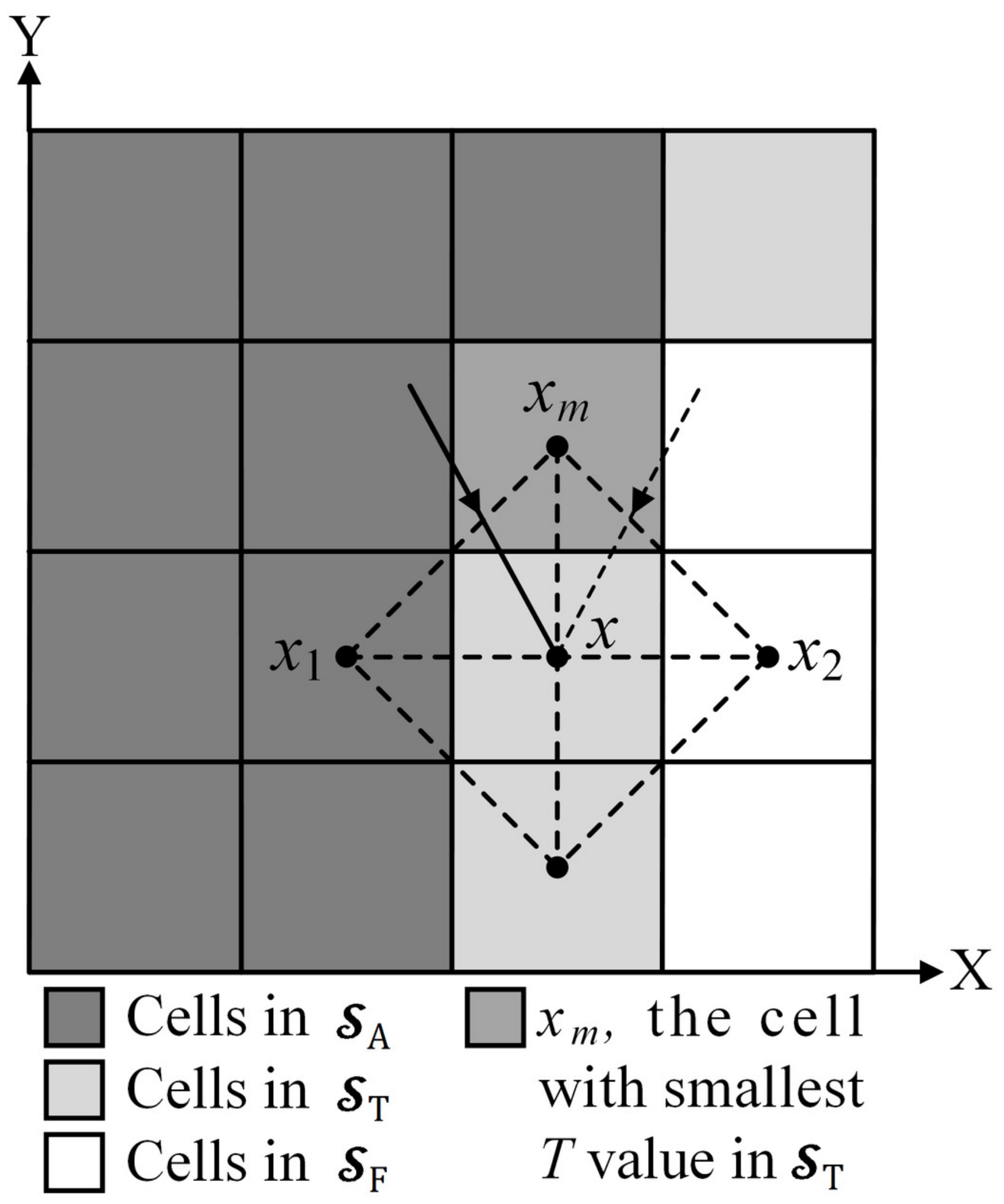




\section{Figure 2}

Maps used or generated in the process of using the $\mathrm{FM}^{2}$ method.

(A) Binary grid map of an obstacle environment. The black cells are obstacle cells, and the white cells are free cells. (B) Arrival time map expanding from the obstacles, which is proportional to the distance from the obstacles. (C) Speed map with a saturated value. The black areas ( 0 value) represent the obstacles, while the white areas (1 value) represent the areas with velocity saturation. The other values are rescaled to better demonstrate the trend.

(D) Arrival time map obtained by applying the $\mathrm{FM}^{2}$ method with $x_{\mathrm{g}}=(50,40)$. The path can be acquired by using the gradient descent method based on this map. 

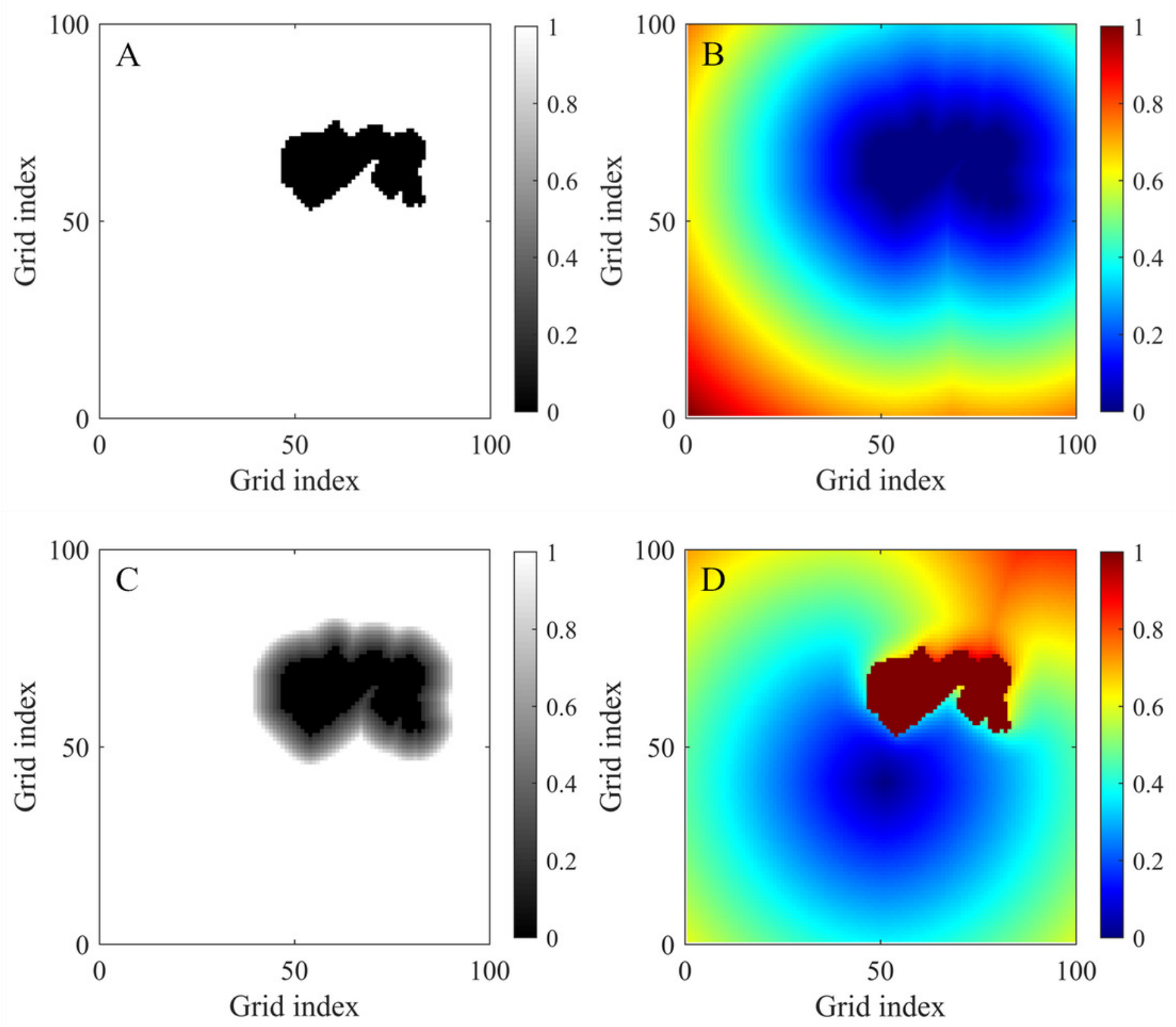
Figure 3

Modified gradient of a path waypoint calculated by the bilinear interpolation method.

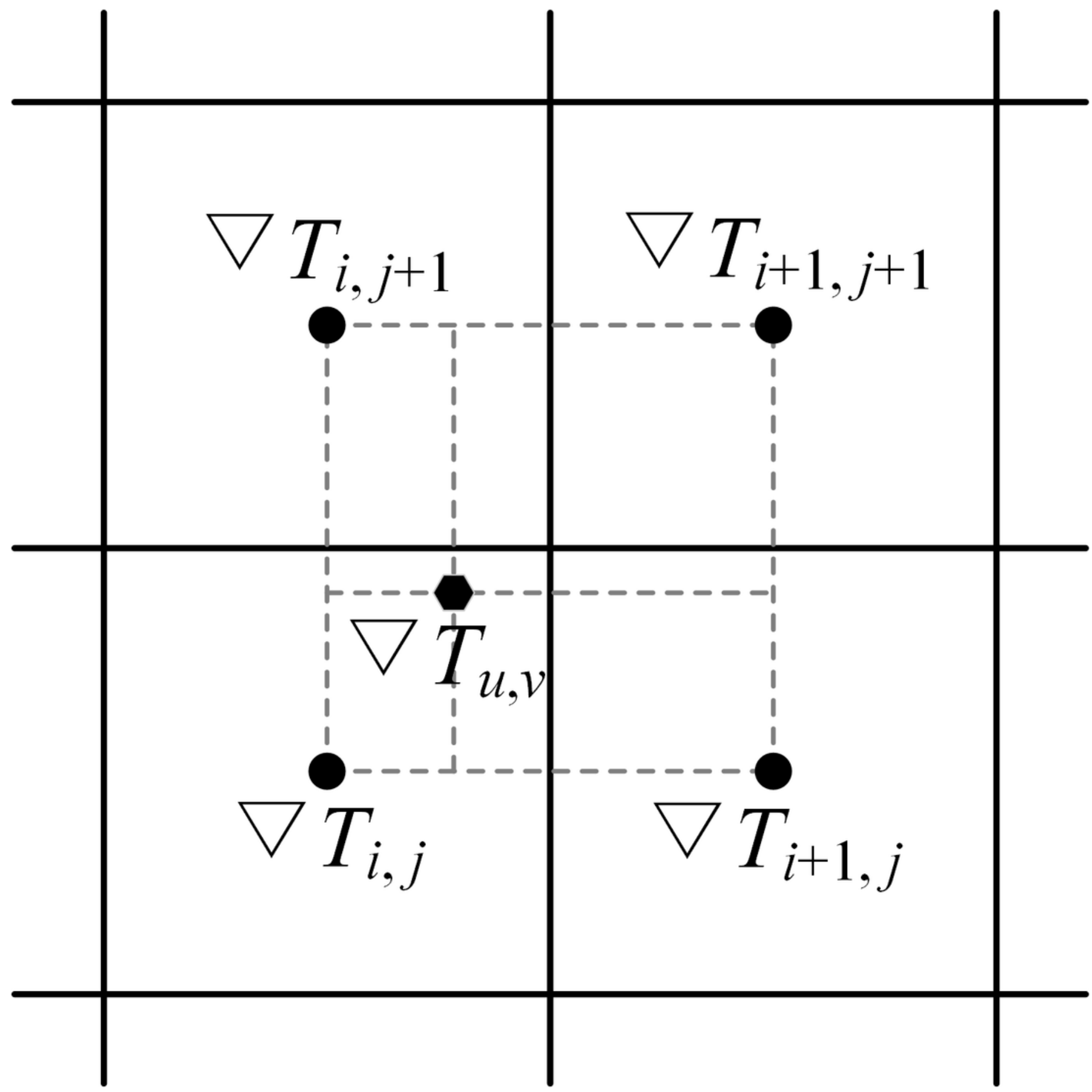


Figure 4

Mapping between an LSR grid map and an HSR grid map.

Here, a case with $L=4$ is shown. $\left(i_{H_{0}}, j_{H_{0}}\right)$ is adaptively fine-tuned based on $x_{g}$. Therefore, $x_{\mathrm{g}}$ is set as the center ( $L$ is odd) or the quasi-center ( $L$ is even) cell of an HSR cell sub-block. 


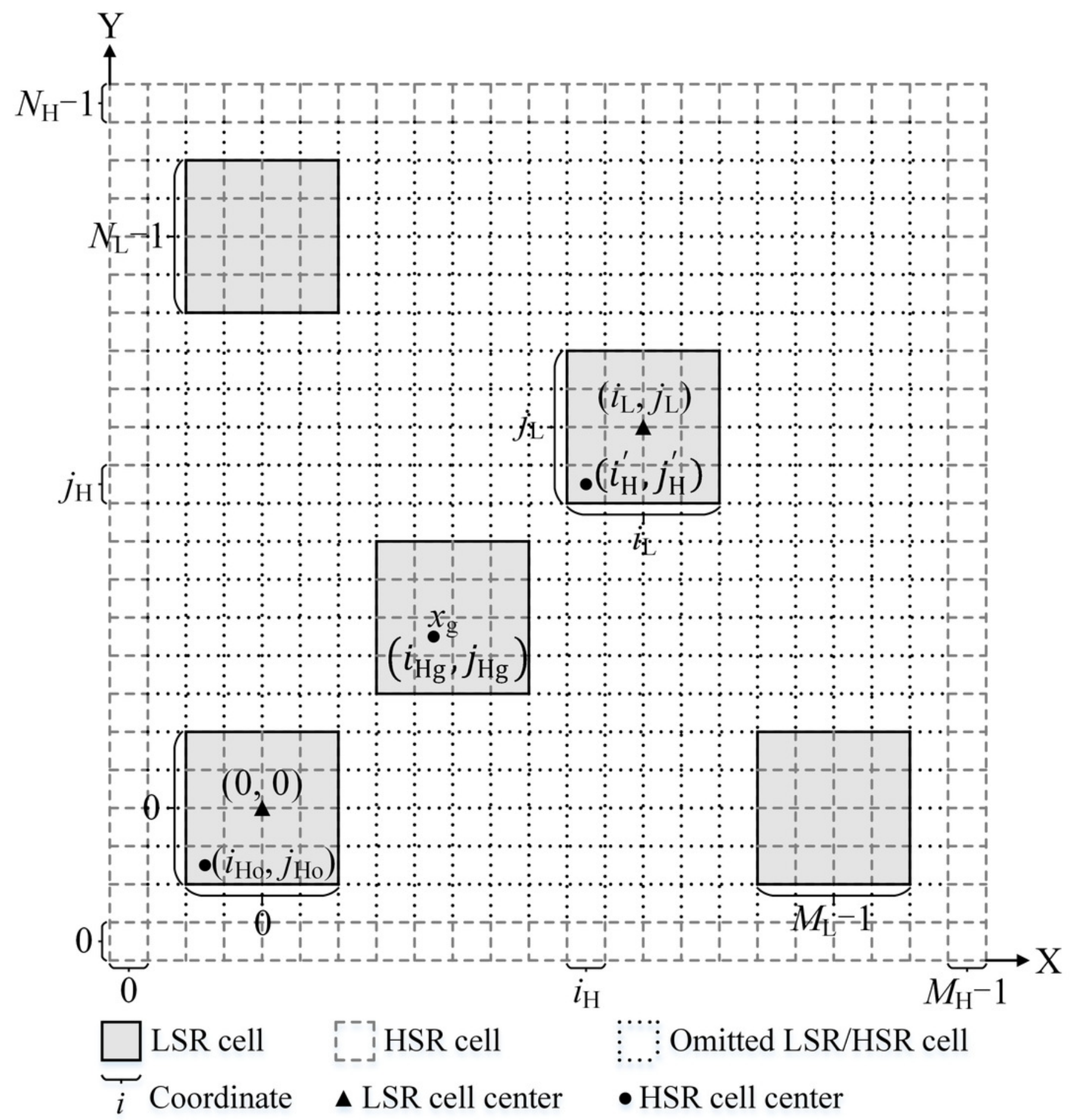




\section{Figure 5}

Influence of $w_{\mathrm{wc}}$ and $w_{\mathrm{sc}}$ on the path.

(A) Influence of $w_{w c}$ on the path. The black region is the obstacle, while the regions with different grayscales from dark to light are the danger region, $S_{d}$; the strong constraint region, $S_{s c}$; and the weak constraint region, $S_{\mathrm{wc}}$ (B) Influence of $w_{\mathrm{sc}}$ on the path.
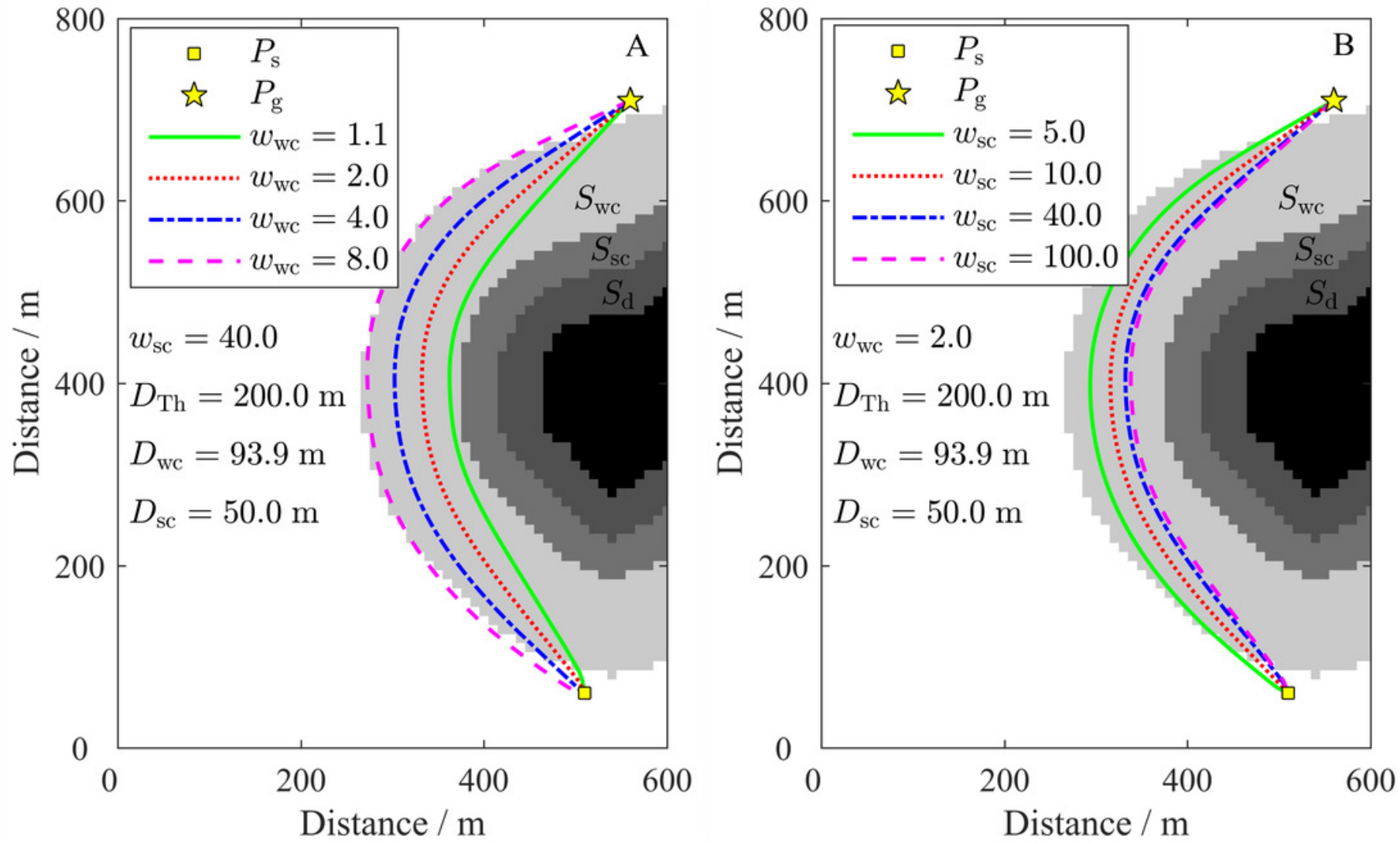
Figure 6

Effective region within the LSR grid map.

The case shown uses $k=4$, and $P_{\text {ff,i }}=\left(f_{\text {floor }}\left(X_{i}\right), f_{\text {floor }}\left(y_{i}\right)\right), P_{\mathrm{cf}, i}=\left(f_{\text {ceil }}\left(x_{i}\right), f_{\text {floor }}\left(y_{i}\right)\right), P_{\mathrm{cc}, i}=\left(f_{\text {ceil }}\left(X_{i}\right)\right.$, $\left.f_{\text {ceil }}\left(y_{i}\right)\right)$, and $P_{\mathrm{fc}, i}=\left(f_{\text {floor }}\left(x_{i}\right), f_{\text {ceil }}\left(y_{i}\right)\right)$ are the four neighbor cells of $P_{i} \cdot P_{\mathrm{ff}, i}$ is the "path-passed" cell with respect to $P_{i}$ in this case.

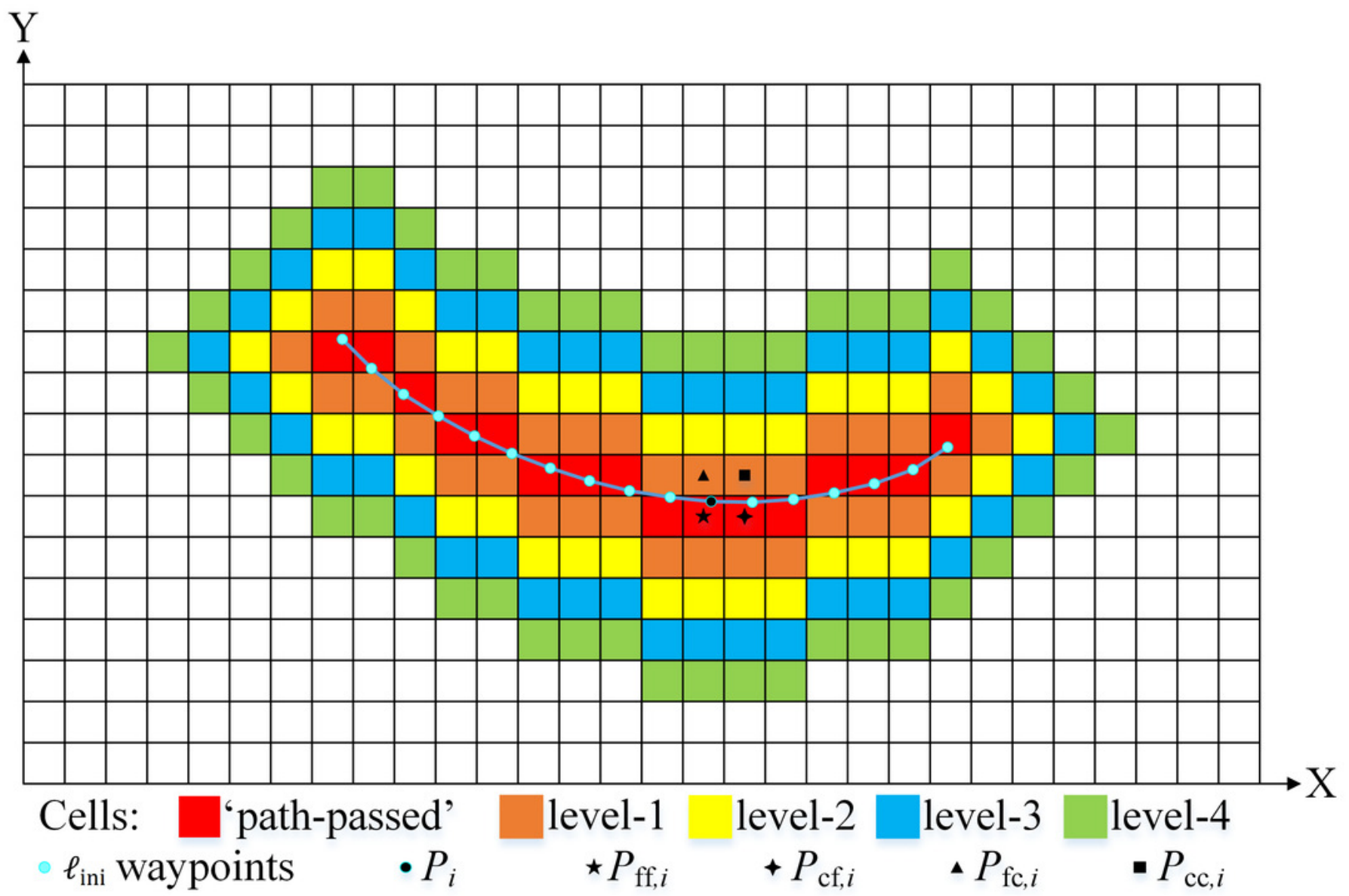




\section{Figure 7}

Distances between the corresponding waypoints of $I_{\text {ref }}$ and $I_{k}(k=3, \ldots, 6)$.

$I_{\text {ref }}$ is the path obtained by applying the IDC-FM² method based on the single HSR grid map directly, while $I_{k}$ is the path obtained by applying the proposed algorithm based on two-level SR grid maps. 

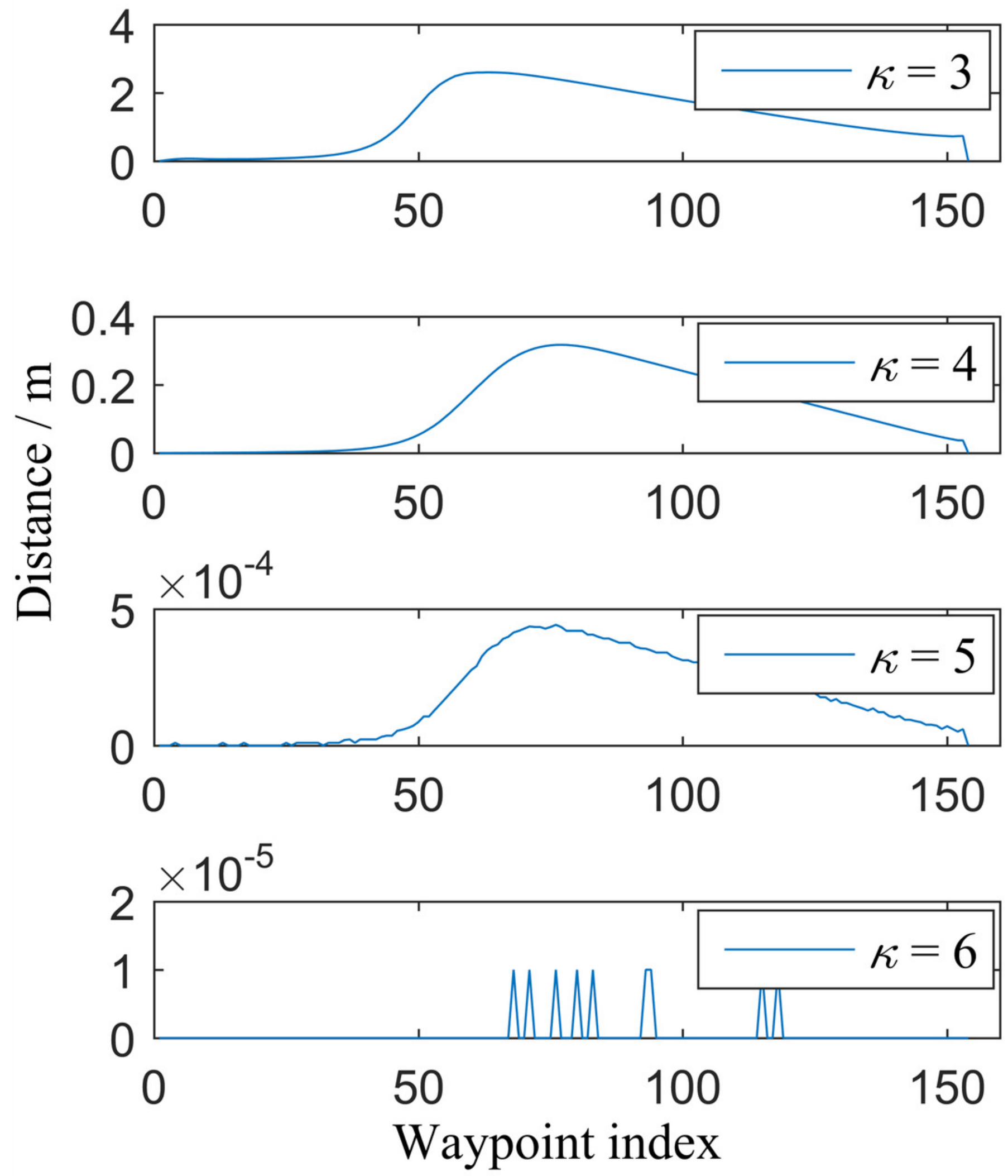


\section{Figure 8}

Algorithm flow of the basic algorithm applying the IDC-FM² method on a single grid map.

This flow is expressed in the form of main data stream and used methods. 


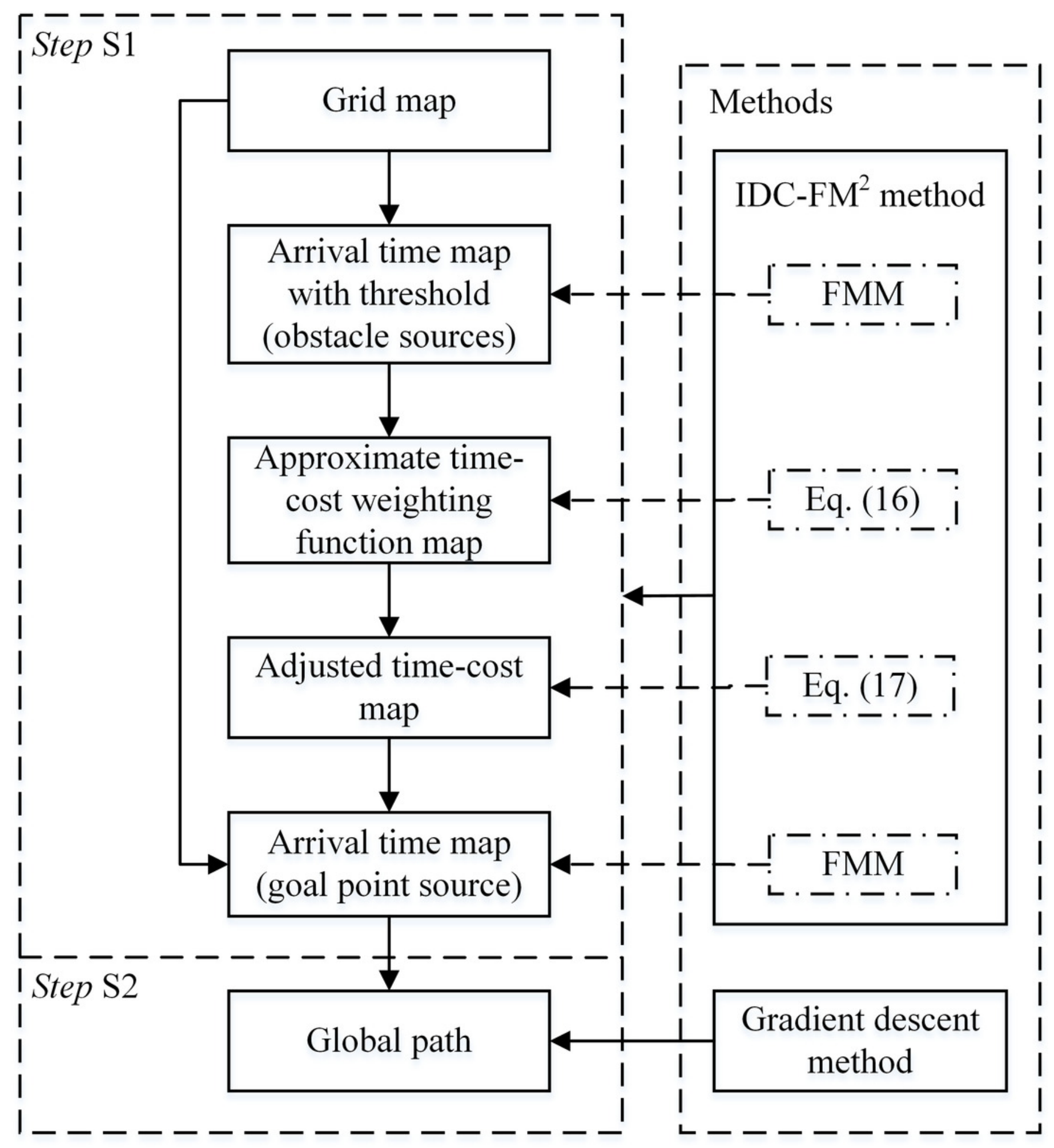




\section{Figure 9}

Algorithm flow of the proposed algorithm performed on two-level SR grid maps.

This flow is expressed in the form of main data stream and used methods. 


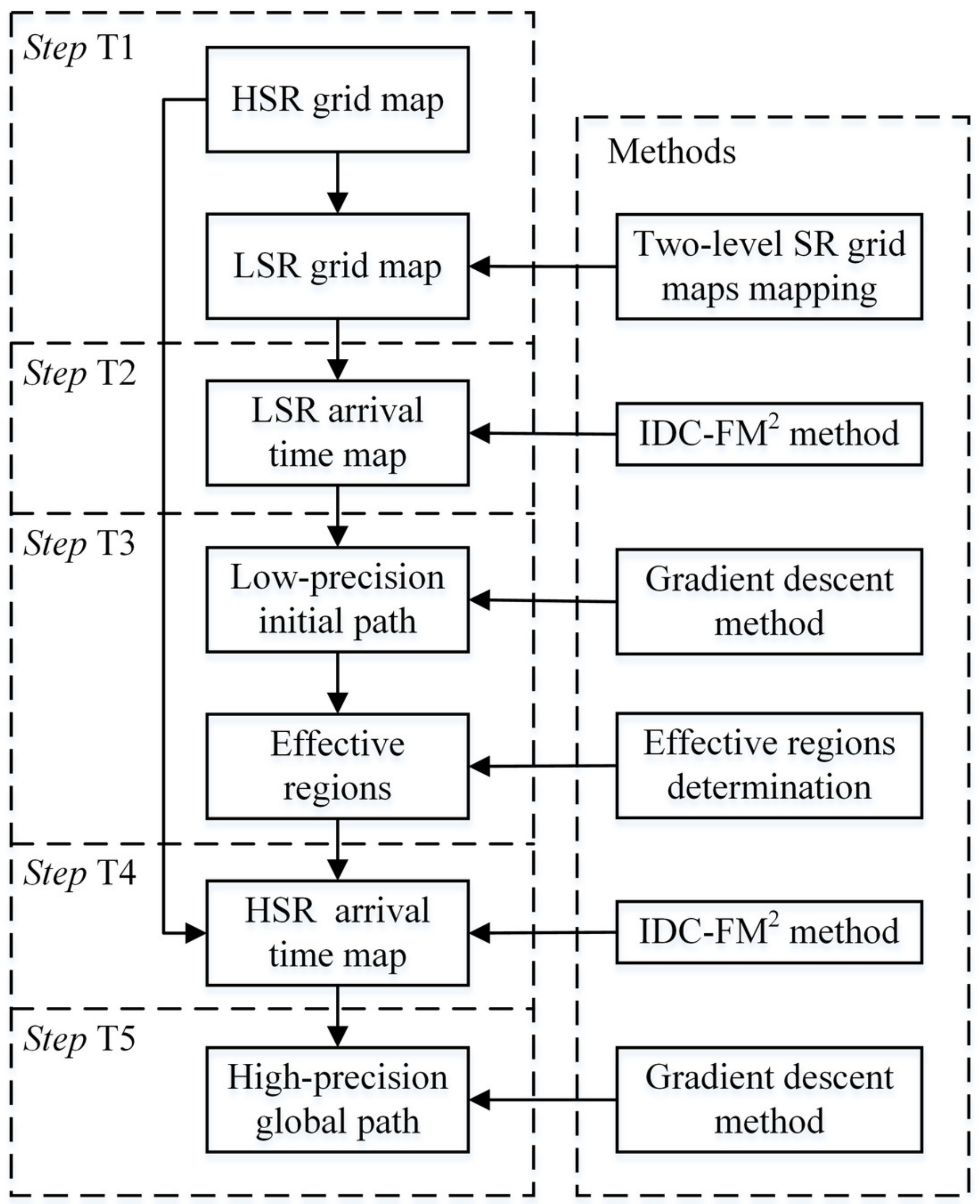




\section{Figure 10}

Google satellite images of the simulation environments.

(A) Surrounding areas of Zhucha Island in Qingdao, Shandong Province, China. Map data @ 2020 Google. (B) Surrounding areas of Changhai County in Liaoning Province, China. Map data @ 2020 Google.

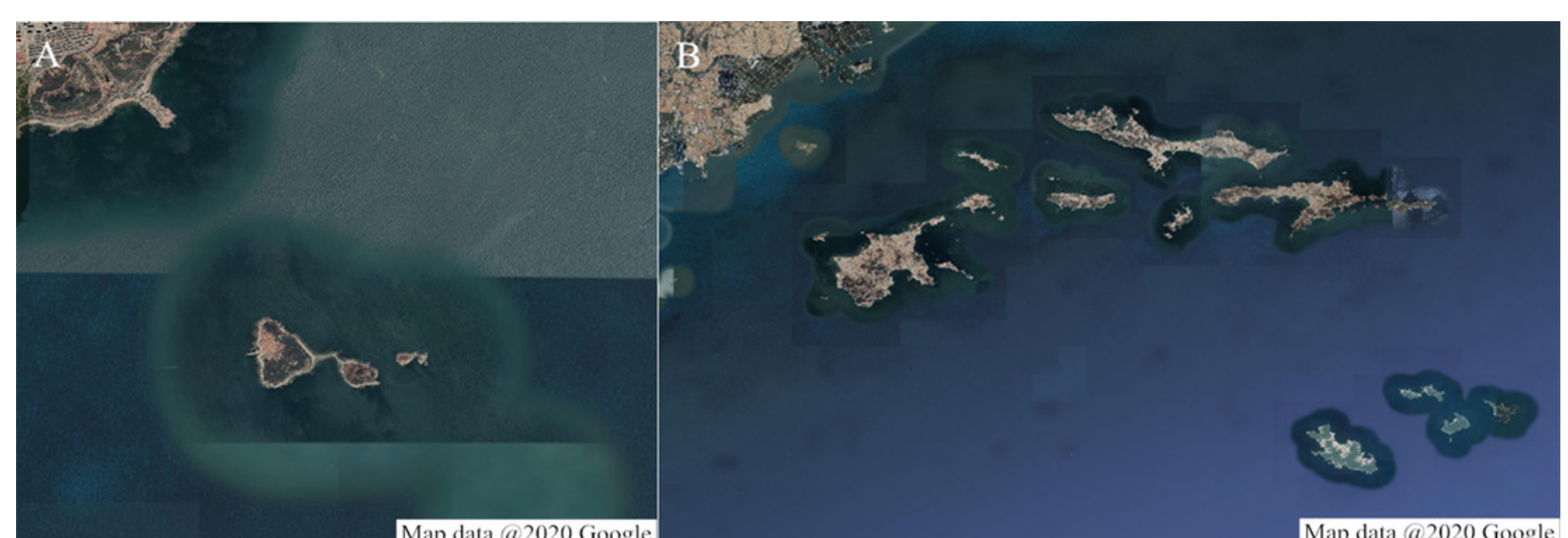


Figure 11

Binary grid maps of the simulation environments.

(A) Binary grid map of the environment shown in Fig. 10A. The black cells indicate obstacle areas, while the white cells indicate the navigable areas for USVs. (B) Binary grid map of the environment shown in Fig. 10B.
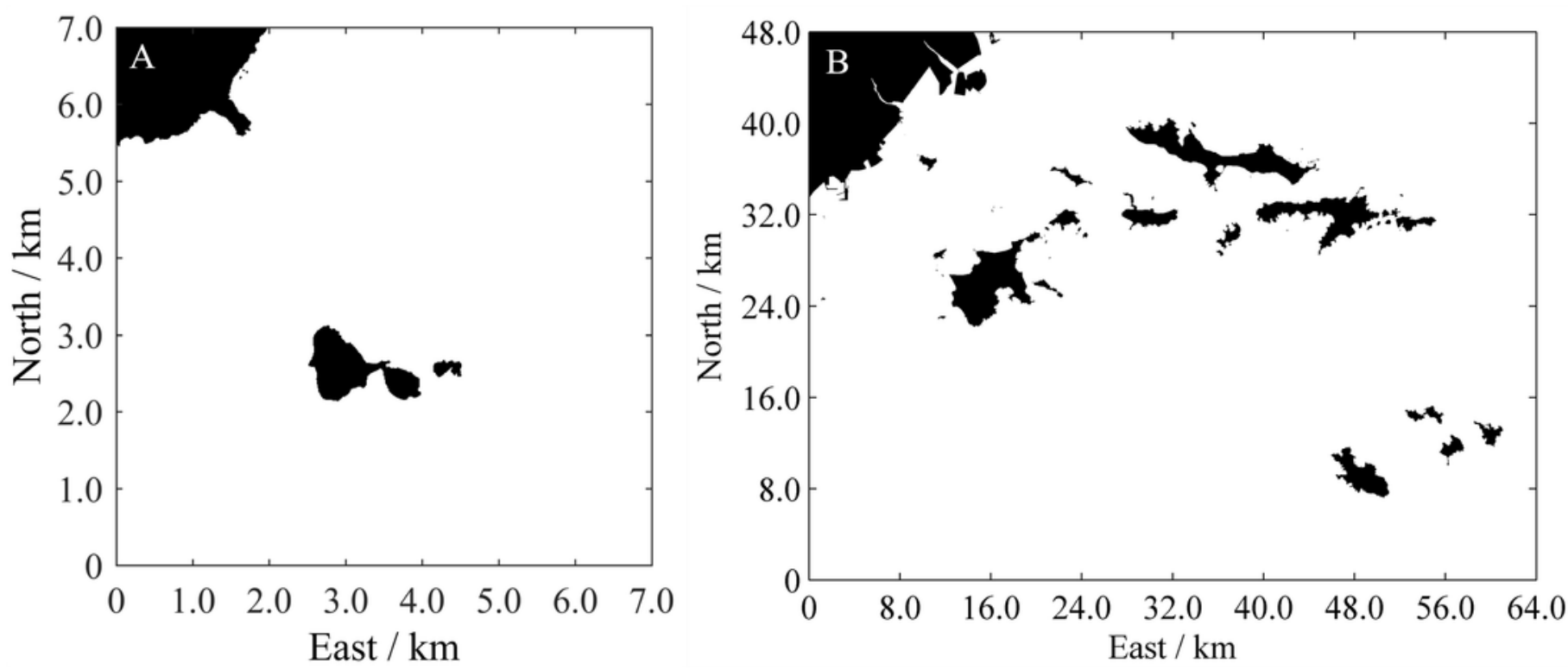


\section{Figure 12}

Path based on the basic FMM $\left(I_{\mathrm{FMM}}\right)$ and paths based on the proposed algorithm with different parameter settings of $w_{x}\left(I_{1}\right.$ to $\left.I_{4}\right)$.

The indicated distances are the estimated quasi-closest distances between the islands and path segments.

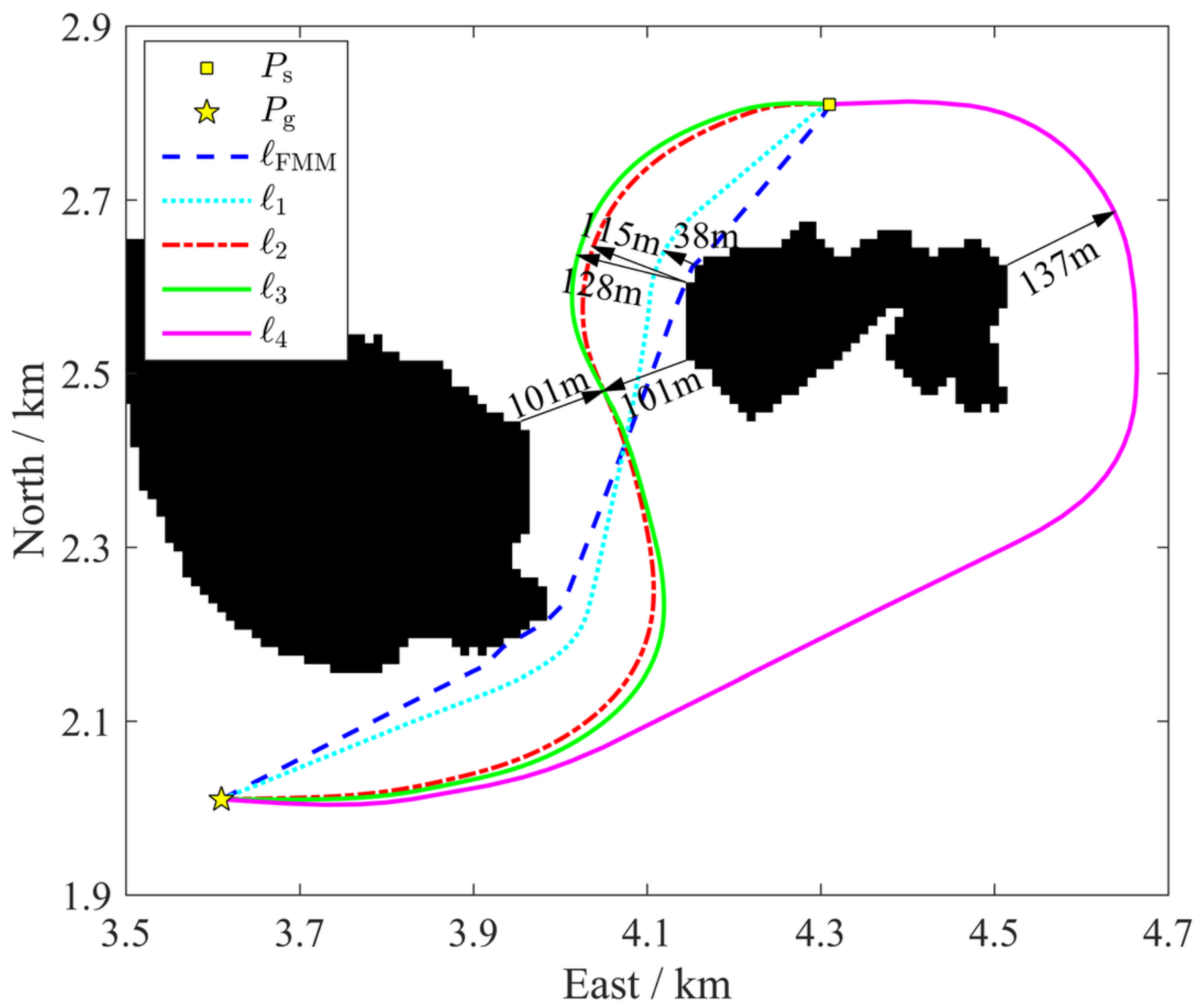




\section{Figure 13}

Paths planned by a classical approach applying the FMM within the map with inflated obstacles and the IDC-FM² method.

When using the classical approach, the obstacle is inflated by $94.0 \mathrm{~m}$. The black cells indicate the raw obstacle, the white cells indicate the free areas, while the cells with different gray scales indicate the inflated obstacles.

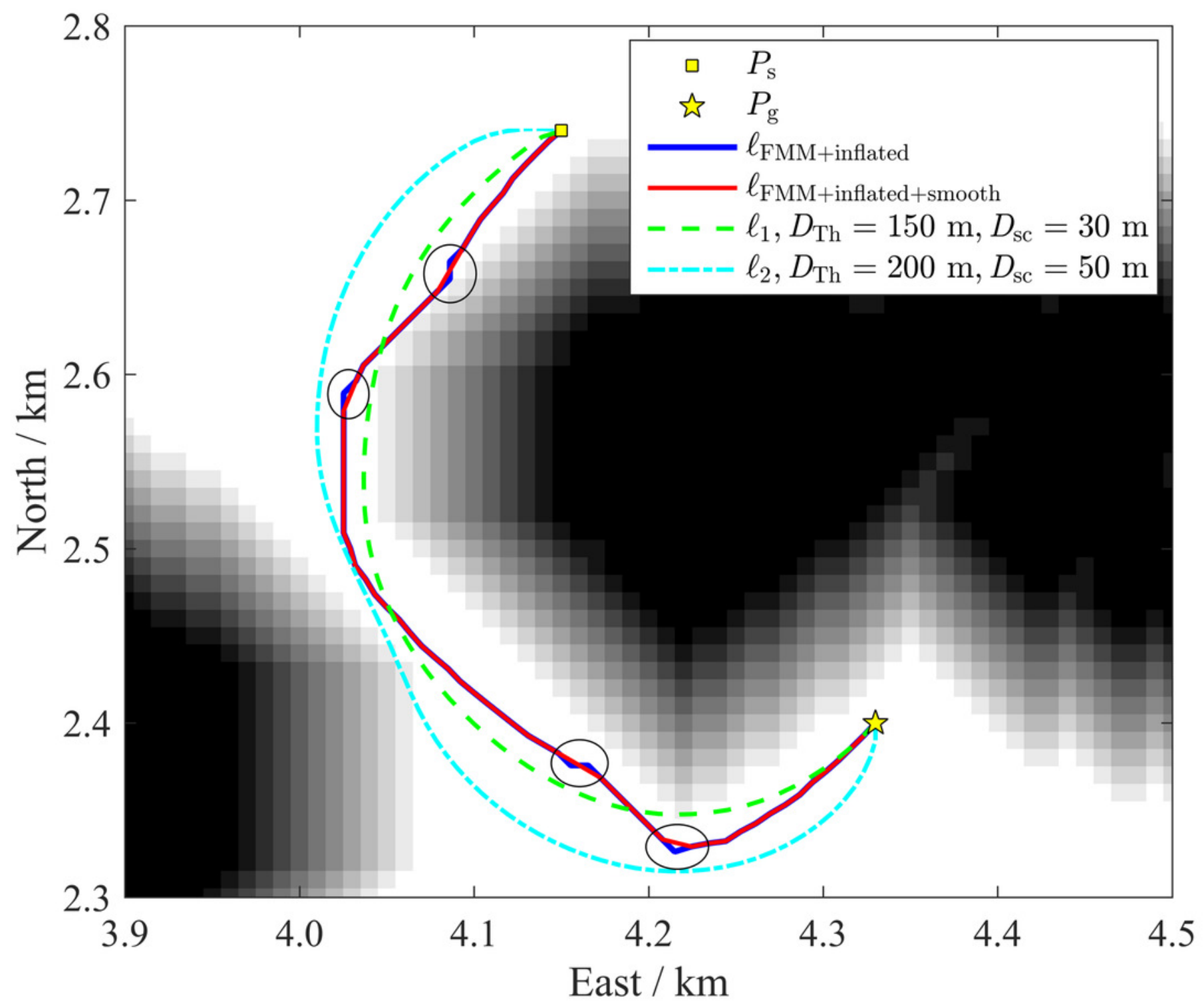


Figure 14

Five typical paths in the surrounding area of Changhai County.

Several portions of these paths marked by rectangles are amplified to show more details in Figure 15.

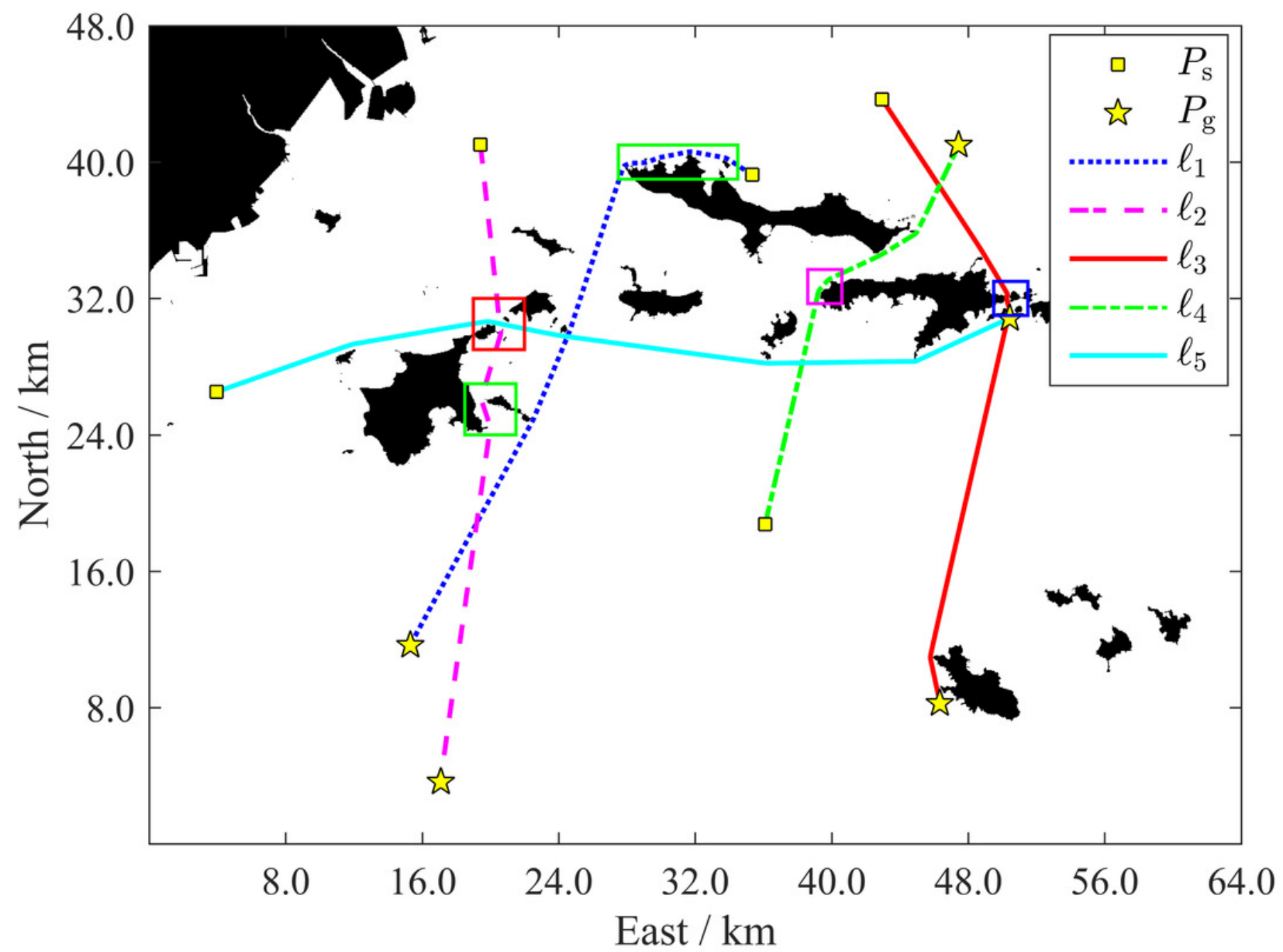




\section{Figure 15}

Enlarged views of path portions.

(A)-(E) show the portions that are indicated by rectangles in Fig. 14. Detailed information can

be acquired from (A) to (E). For example, all paths maintain a relatively safe distance from each island, the paths are smooth even when they turn around islands, and paths across narrow channels can be planned along the quasi-midline of the channels. 

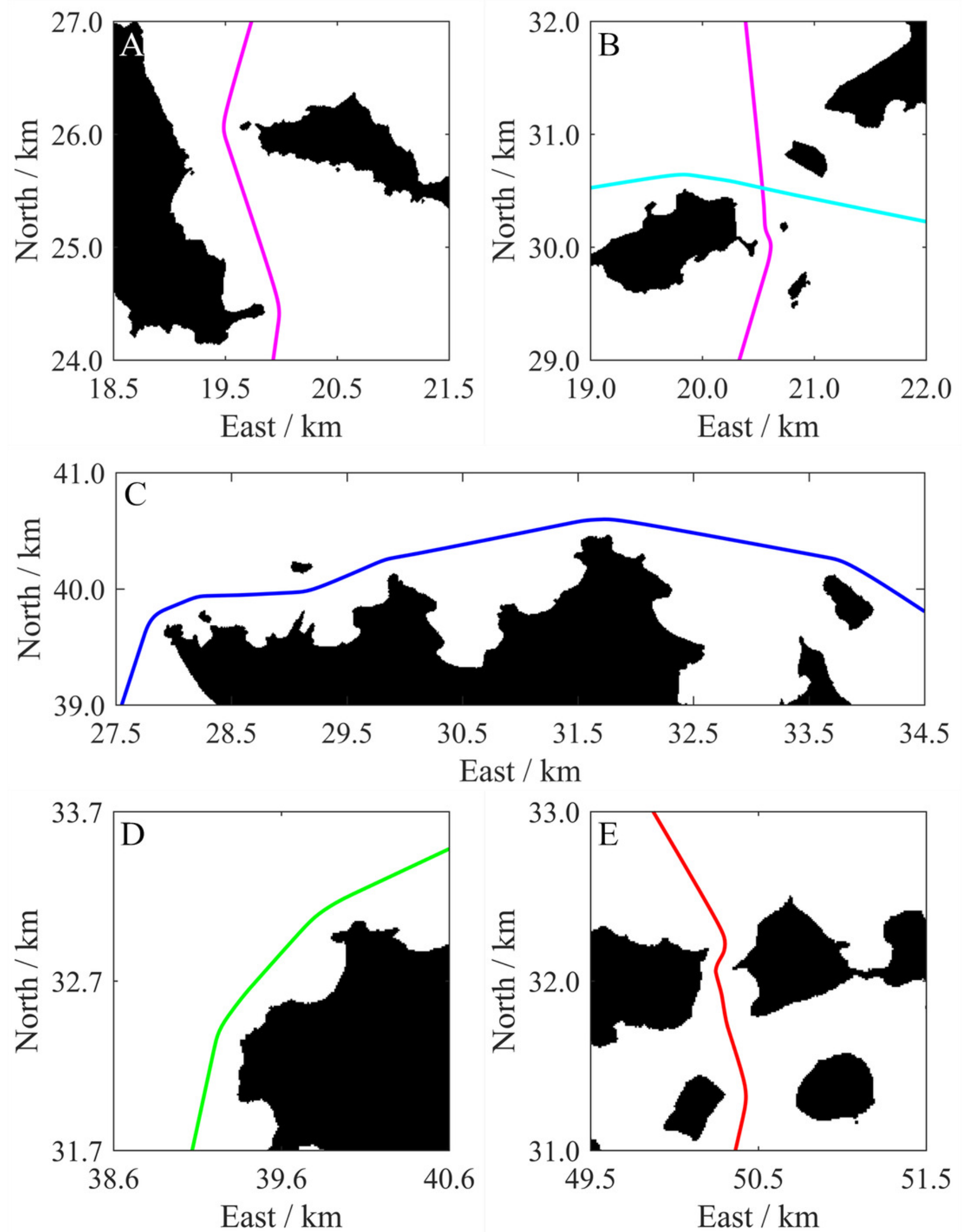

Peer] Comput. Sci. reviewing PDF | (CS-2021:02:58122:2:0:NEW 27 May 2021) 